\title{
Scaling Analysis of Thermodynamic Properties in the Critical Region of Fluids
}

\author{
M. Vicentini-Missoni, * J. M. H. Levelt Sengers, and M. S. Green** \\ Institute for Basic Standards, National Bureau of Standards, Washington, D.C. 20234
}

(July 24, 1969)

\begin{abstract}
A review of the scaled equation of state proposed for the critical region of fluids and magnets is given using the language appropriate for fluids. The experimental evidence for the validity of the basic hypothesis underlying this equation of state is discussed in detail. Experimental data in the critical regions of $\mathrm{CO}_{2}, \mathrm{Xe}$, and $\mathrm{He}^{4}$ are then analyzed using a closed-form expression for the chemical potential as a function of density and temperature, based on scaling ideas. Agreement between the proposed equation and the experimental dat is found for the three substances. The results of the scaling of $\Delta \mu, \Delta \rho, t$ data are shown not to be in contradiction with the analysis, also based on scaling ideas, of independent experimental measurements of both specific heat and vapor pressure.
\end{abstract}

Key words: $\mathrm{CO}_{2}$; chemical potential; critical point parameters; critical region; fluids; $\mathrm{He}^{4}$; scaling laws; specific heat; thermodynamic properties; vapor pressure; xenon.

\section{Introduction}

The thermodynamic anomalies in the critical region are usually described by means of power laws, assumed to be asymptotically valid approaching the critical point. Those of direct interest to us are, in Fisher's notation $[1]^{1}$ :

Coexistence curve: $\Delta \rho \simeq B(-t)^{\beta}$

Critical isotherm:

$$
\begin{aligned}
\left|p\left(\rho, T_{c}\right)-p\left(\rho_{c}, T_{c}\right)\right| \sim \mid \mu(\rho, & \left.T_{c}\right)-\mu\left(\rho_{c}, T_{c}\right) \mid \\
& \simeq \Delta \cdot \Delta \rho|\Delta \rho|^{\delta-1}
\end{aligned}
$$

Compressibility:

on the critical isochore, $K_{T} \simeq \Gamma t^{-\gamma}, T>T_{c}$

along the coexistence curve in the one-phase region:

$$
\rho^{2} K_{T} \simeq \Gamma^{\prime}(-t)^{-\gamma} \quad T<T_{c}
$$

Specific heat at constant volume:

on the critical isochore,

$$
\begin{array}{ll}
C_{v} \simeq\left(A^{+} / \alpha\right) t^{-\alpha} & T>T_{c} \\
C_{v} \simeq\left(A_{\mathrm{II}}-\alpha^{\prime}\right)(-t)^{-\alpha^{\prime}} & T<T_{c}
\end{array}
$$

along the coexistence curve in the one-phase region:

$$
\rho C_{v} \simeq\left(A_{\mathrm{I}}^{-} / \alpha^{\prime}\right)(-t)^{-\alpha^{\prime}} \quad T<T_{c}
$$

* On leave of absence from the University of Rome, Rome, Italy.

**Present address: Temple University, Philadelphia, Penn. 19122

' Figures in brackets indicate the literature references at the end of this paper.
Here $\Delta \rho=\left(\rho-\rho_{c}\right) / \rho_{c}, t=\left(T-T_{c}\right) / T_{c}$ and all properties are measured in reduced units through the appropriate combinations of critical parameters. Thus the temperature is measured in units $T_{c}$, the density in units $\rho_{c}$, the chemical potential $\mu(\rho, T)$ in units $p_{c} / \rho_{c}$, the specific heat per unit volume $\rho C_{c}$ in units $p_{c} / T_{c}$, etc. The critical density, pressure and temperature are therefore unity by definition.

The scaled equation-of-state, recently proposed $[2,3,4]$, is a partial formulation of the thermodynamic behavior in the critical region, incorporating these anomalies. In this paper we discuss the critical region of fluids in the light of this theory. A review of the basic hypothesis and the formulation of the scaling laws for fluids is given in section 2. The experimental evidence for the validity of the basic hypothesis is discussed in detail in section 3. Our previous tests [5] of the extent to which experimental data on fluids possess the scaling property are recalled, and the points where the early analysis needs improvement are discussed (sec. 4). An explicit expression for the equation-of-state in terms of scaled variables is proposed in section 5. The details of the procedure followed in analyzing the experimental data are given in section 6. The results of the analysis of the data, complete with internal and external consistency checks, are reported in sections 7 to 9 . Finally, conclusions are presented in section 10 .

\section{Scaled Equation-of-State}

The equilibrium thermodynamic properties of a fluid are determined by the knowledge of either the Helm- 
holtz free energy per unit volume $a(\rho, T)$ as a function of density $\rho$ and temperature $T$, or of the corresponding free energy per mole $A(v, T)$ as a function of molar volume $v$ and temperature. In the first case the variable conjugate to the density is the chemical potential $\mu=(\partial a / \partial \rho)_{T}$ while in the second case the variable conjugate to the molar volume is the pressure $p=-(\partial A / \partial v)_{T}$. It is a question of which pair of conjugate variables is most suitable for discussing thermodynamic properties in the critical region. We have made the choice on the basis of symmetry arguments discussed more fully in the next section. Magnetic critical phenomena show, by definition, complete symmetry with respect to the line $M=0$. Thus, the spontaneous magnetization is fully symmetric around $M=0$. Since the analogy of magnetic and fluid critical phenomena plays such a basic role in scaling law theory, we choose the pair of variables which shows the best symmetry around the critical isochore. It is well known that for simple fluids the phase boundary shows more symmetry around $\rho_{c}$ in the $\rho T$ plane than around $v_{c}$ in the $v T$ plane. This and other symmetry considerations (sec. 3) lead to a preference for the pair $\mu, \rho$.

Widom [2], using chemical potential, density and temperature as variables, first proposed an equationof-state for fluids; it is exactly equivalent to those proposed for the Ising model and for magnets $[3,4]$, as was shown by Griffiths [4]. The main assumptions on which this equation is based are the plausible ones that free energy and chemical potential are regular in $\rho$ and $t$ throughout the entire one-phase region with possible exclusion of the phase boundary. Moreover, the quantity $\Delta \mu=\mu(\rho, t)-\mu\left(\rho_{c}, t\right)$ is also assumed to be regular throughout the entire one-phase region, and, at least asymptotically, antisymmetric around $\rho_{c}$. It should be emphasized that regularity of $\mu$ and $\Delta \mu$ implies regularity of $\mu\left(\rho_{c}, t\right)$ at the critical temperature, as is the case in the lattice gas.

In the following, a review of the properties of the equation-of-state is given according to Griffiths' formulation [4]; then, the derived properties of the free energy and the specific heat are examined.

\subsection{The Equation-of-State}

The asymptotic equation for the phase boundary, eq (1.1), suggests the introduction of a variable $x$ defined by

$$
x=t /|\Delta \rho|^{1 / \beta} .
$$

In terms of this variable, it is hypothesized that the equation of state is of the form:

$$
\Delta \mu=\Delta \rho|\Delta \rho|^{\delta-1} h(x) .
$$

The quantity $h(x)$ is a function of the variable $x$ only, which according to the requirements of analyticity of $a(\rho, t), \mu(\rho, t)$, and $\Delta \mu(\rho, t)$ must have the following properties:

(1) It is a real positive function of $x$ everywhere in the range $-x_{0}<x<\infty$ which vanishes at the phase boundary $x=-x_{0}, x_{0}=B^{-1 / \beta}$, with a finite slope:

$$
\left(\frac{\partial \mu}{\partial \rho}\right)_{\text {coex. }}=\left(\rho^{2} K_{T}\right)_{\text {गех }}^{-1}=\beta^{-1} h^{\prime}\left(-x_{0}\right) r_{0}^{1-\beta(\delta-1)} t^{\beta(\delta-1)}
$$

and from the definition given in eq (1.3)

$$
\begin{aligned}
& \gamma^{\prime}=\beta(\delta-1) \\
& \Gamma^{\prime}=\left[\beta^{-1} h^{\prime}\left(-x_{0}\right) x_{0}^{1-\gamma^{\prime}}\right]^{-1} .
\end{aligned}
$$

About $x=0 h(x)$ possesses the series expansion

$$
h(x)=\sum_{j=0}^{x} h_{j} x^{j}=h_{0}+h_{1} x+h_{2} x^{2}+\ldots
$$

so that on the critical isotherm $|\Delta \mu|=h_{0}|\Delta \rho|^{\delta}$ and from the definition in eq (1.2)

$$
\Delta=h_{0}
$$

(2) For large $x, h(x)$ has the series expansion

$$
h(x)=\sum_{n=1}^{\infty} \eta_{n} x^{\beta(\delta+1-2 n)}
$$

which means that

$$
\left(\frac{\partial \mu}{\partial \rho}\right)_{\rho=\rho_{c}}=\left[K_{T}\right]_{\rho=\rho_{c}}^{-1}=\eta_{1} t^{\beta(\delta-1)}
$$

and

$$
\begin{gathered}
\gamma=\beta(\delta-1)=\gamma^{\prime} \\
\Gamma=\eta_{1}^{-1}
\end{gathered}
$$

(3) The compressibility is given in general by

$$
\left[\rho^{2} K_{T}\right]^{-1}=|\Delta \rho|^{\delta-1}\left[\delta h(x)-\frac{1}{\beta} x h^{\prime}(x)\right] .
$$

For it to be nonnegative $h(x)$ must satisfy the condition

$$
\beta \delta h(x) \geqslant x h^{\prime}(x) .
$$

Thus, with the definition $\gamma=\beta(\delta-1)$, the scaling law eq $(2.2)$ is consistent with anomalies $(1.1,1.2$, 1.3).

\subsection{The Free Energy}

The free energy per unit volume $a(\rho, t)$ is only partially determined by the equation of state: $\mu=\mu(\rho, t)=(\partial a / \partial \rho)_{t}$. First, since the scaling law equation is an expression for $\Delta \mu$, an unknown function $\mu\left(\rho_{c}, t\right)$ multiplied by $\rho$ enters into the expression for $a(\rho, t)$. Secondly, an integration constant $A_{0}(T)$ will occur additively. It is assumed that $A_{0}(T)$ is an analytic function of the temperature.

In the general case $\alpha \neq 0(0<\alpha=2-\beta(\delta+1)<1)$ we have the following expression for the free energy: 


$$
a(\rho, t)=A_{0}(T)+\rho \mu\left(\rho_{c}, t\right)+|\Delta \rho|^{\delta+1} a_{\alpha}(x)
$$

with $a_{\alpha}(x)$ related to $h(x)$ through:

$$
-x a_{\alpha}^{\prime}(x)+(2-\alpha) a_{\alpha}(x)=\beta h(x)
$$

$a_{\alpha}(x)$ is analytic everywhere for $-x_{0}<x<\infty$; near $x=0$ it can be written in the form

$$
\begin{aligned}
& a_{\alpha}(x)=\beta\left\{\frac{h_{0}}{2-\alpha}+\frac{h_{1} x}{1-\alpha}\right. \\
& \left.-x|x|^{1-\alpha} \int_{0}^{x}\left[h(y)-h_{1} y-h_{0}\right]|y|^{\alpha-3} d y\right\} .
\end{aligned}
$$

In the two-phase region $a_{\alpha}(x)$ is constant and equal to its value on the phase boundary $a_{\alpha}\left(-x_{0}\right)$. (The numerical value of $a_{\alpha}\left(-x_{0}\right)$ can be evaluated by using in eq (2.13) the series expansion for $h(y)$ near $y=0$ with as many terms as necessary to cover the range $\left.-x_{0}, 0\right)$.

For large positive $x, a_{\alpha}(x)$ can be expanded as

$$
a_{\alpha}(x)=C x^{2-\alpha}+\frac{1}{2} \sum_{n=1}^{\infty} n^{-1} \eta_{n} x^{2-\alpha-2 \beta n}
$$

the constant $C$ being given by

$$
C=-\beta \int_{0}^{\infty}\left[h(y)-h_{0}-h_{1} y\right] y^{\alpha-3} d y .
$$

In the case $\alpha=0$, the free energy is given by

$$
\begin{aligned}
a(\Delta \rho, t)=A_{0}(T)+\mu\left(\rho_{c}, t\right) \rho & +|\Delta \rho|^{\delta+1} a_{0}(x) \\
& +h_{2} t^{2} \ln |\Delta \rho|
\end{aligned}
$$

with $a_{0}(x)$ satisfying the differential equation

$$
-x a_{0}^{\prime}(x)+2 \alpha_{0}(x)=\beta\left[h(x)-h_{2} x^{2}\right] .
$$

The necessary and sufficient condition for the heat capacity to be positive is that $a_{\alpha}^{\prime \prime}(x) \leqslant 0$ for all $x$ in the range $-x_{0}<x<\infty$. This is hard to translate into any simple condition on $h(x)$ or its derivatives. However, the condition that for all $x$ in the range $-x_{0}<x<\infty$ $h^{\prime \prime}(x)$ be nonnegative is sufficient for the positivity of the heat capacity and is useful in constructing functions.

From the thermodynamic relation $p=\mu \rho-a$, through eq $(2.2,2.11)$, the equation of state in the variables $p, \Delta \rho, t$ may be derived:

$$
\begin{gathered}
p(\rho, T)=\Delta \rho|\Delta \rho|^{\delta-1} h(x)+|\Delta \rho|^{\delta+1}\left[h(x)-a_{\alpha}(x)\right] \\
-A_{0}(T) .
\end{gathered}
$$

The behavior of the vapor pressure $p_{v}(t)$ and the pressure above $T_{c}$ on the critical isochore will then be given, respectively, by

$$
\begin{gathered}
p_{r}(T)=-A_{0}(T)-a_{\alpha}\left(-x_{0}\right) x_{0}^{\alpha-2}(-t)^{2-\alpha}, t<0 \\
p\left(\rho_{c}, t\right)=-A_{0}(T)-C t^{2-\alpha}, t>0 .
\end{gathered}
$$

The nonanalytic term is of the form $|t|^{2-\alpha}$. Its coefficients for positive $t$ differs from that for negative $t$. However both coefficients are related to $h(x)$, through eq (2.13) and (2.15) respectively. They are both positive since $a_{\alpha}\left(-x_{0}\right)$ and $C$ are negative. Since the chemical potential along the critical isochore is assumed non singular at $t=0$, the singularity in the pressure is directly related to the singularity in the specific heat according to the scaling laws. Indeed the second derivative of the pressure on the critical isochore according to eq (2.19) diverges with the exponent $\alpha$ and the same exponent is found for the specific heat. From

$$
\rho C_{c}=-T\left(\frac{\partial^{2} a}{\partial t^{2}}\right)_{\rho}
$$

( $T$ in units $T_{c}, \rho C_{v}$ in units $p_{c} / T_{c}$ )

the scaling equations for the specific heat are derived to be:

$$
\begin{aligned}
-\rho C_{c} / T=\frac{d^{2} A_{0}}{d t^{2}}+\frac{d^{2} \mu\left(\rho_{c}, t\right)}{d t^{2}}+\Delta \rho \frac{d^{2} \mu\left(\rho_{c}, t\right)}{d t^{2}}- & \\
& +a\left(-x_{0}\right) x_{0}^{\alpha-2}(2-\alpha)(1-\alpha)(-t)^{-\alpha}
\end{aligned}
$$$$
\text { two-phase region }
$$

$$
\begin{aligned}
\left.-\rho C_{v} / T=\frac{d^{2} A_{0}}{d t^{2}}+\frac{d^{2} \mu\left(\rho_{c}, t\right)}{d t^{2}}-t\right) \frac{d^{2} \mu\left(\rho_{c}, t\right)}{d t^{2}} & \\
& +|\Delta \rho|^{-\alpha / \beta} a_{\alpha}^{\prime \prime}(x)
\end{aligned}
$$

one-phase region.

The singular behavior is described by the term with $(-t)^{-\alpha}$ in the two-phase region and by the term with $|\Delta \rho|^{-\alpha / \beta}$ in the one-phase region. It is therefore derived completely from the properties of $h(x)$; in particular along the critical isochore in the one-phase region:

$$
a_{\alpha}^{\prime \prime}(x)=C(2-\alpha)(1-\alpha) x^{-\alpha} .
$$

Thus $\rho C_{v} / T$ behaves as $t^{-\alpha}$; also on any other isochore $t^{-\alpha}$ is the asymptotic behavior for large $x$. The complete expressions for the specific heat on the critical isochore are:

$$
\begin{aligned}
& -\frac{C_{r}}{T}=\frac{d^{2} A_{0}}{d t^{2}}+\frac{d^{2} \mu\left(\rho_{c}, t\right)}{d t^{2}} \\
& +a_{\alpha}\left(-x_{0}\right) x_{0}^{\alpha-2}(2-\alpha)(1-\alpha)(-t)^{-\alpha}, \quad t<0 \\
& -\frac{C_{r}}{T}=\frac{d^{2} A_{0}}{d t^{2}}+\frac{d^{2} \mu\left(\rho_{c}, t\right)}{d t^{2}}, \\
& \quad+C(2-\alpha)(1-\alpha) t^{-\alpha}, \quad t>0
\end{aligned}
$$

in striking analogy with (2.19). 
The coefficients defined in eq (1.4) are found to be related to the properties of $h(x)$ by:

$$
\begin{gathered}
A^{+} / \alpha=-C(2-\alpha)(1-\alpha)=\beta \int_{0}^{\infty} h^{\prime \prime}(y) y^{\alpha-1} d y \\
A_{\overline{\mathrm{II}}} / \alpha=-(2-\alpha)(1-\alpha) a_{\alpha}\left(-x_{0}\right) x_{0}^{\alpha-2} \\
A_{\mathrm{I}}^{-} / \alpha=\beta \int_{-x_{0}}^{0} h^{\prime \prime}(y)|y|^{\alpha-1} d y
\end{gathered}
$$

while the discontinuity in the specific heat across the phase boundary, $\Delta \rho C_{v}$, is given by

$$
\Delta \rho C_{v}=\beta x_{0}^{\alpha-1} h^{\prime}\left(-x_{0}\right)(-t)^{-\alpha} .
$$

For the case $\alpha=0$, analogous expressions can be derived from a free energy of the form (2.16). In this case the specific heat has a symmetrical logarithmic singularity on the critical isochore with a superimposed finite jump and the pressure at $\rho=\rho_{c}$ has singular terms of the form $a t^{2} \ln |t|+b t^{2}$ with the same value of $a$ but different $b$ depending on whether $T \gtrless 0$. Details are given in appendix I.

\section{Scaling Symmetries and Real Fluids}

\subsection{Antisymmetry of the Chemical Potential}

The scaling laws assume certain symmetries in the thermodynamic variables which are present in magnetic systems, in the Ising model, and therefore in the lattice gas, but cannot a priori be expected to hold in real fluids. In the magnetic case, the spontaneous magnetization is symmetric w.r.t. the line $M=0$, whereas the field is antisymmetric in $M$. For fluids on thermodynamic grounds alone, we have the option to use a $p, v$ or a $\mu, \rho$ analogy to the magnetic case. The lattice gas has a coexistence curve symmetric in the density whereas the chemical potential is antisymmetric with respect to the critical isochore. For real gases, a consideration of the $p, v$ and the $\mu, \rho$ behavior at different temperatures strongly favors the second option.

In figures 1 to 3 , we show the behavior of $\Delta p=p(\rho, t)$ $-p\left(\rho_{c}, t\right)$ versus $\left|v-v_{c}\right|$ and of $|\Delta \mu|$ versus $|\Delta \rho|$ for the gases $\mathrm{CO}_{2}[6]$, Ar [7], and $\mathrm{He}^{4}$ [8]. Above the critical temperature, the chemical potential shows a striking antisymmetry, evidenced by nearcoincidence of the $\Delta \mu(+\Delta \rho)$ and $\Delta \mu(-\Delta \rho)$ curves over a range of densities up to nearly 50 percent from critical, and up to temperatures far above critical. (See figs. 1 and 2 for $\mathrm{CO}_{2}$ and Ar.) This antisymmetry is absent in the $p, v$ representation. Plotting $\Delta p$ versus $\Delta \rho$, approximate antisymmetry is observed in a small density range only. Thus, above $\boldsymbol{T}_{c}$, the choice of variables is unambiguously the pair $\mu, \rho$ from the point of view of symmetry. It may be noted that the practice of analyzing $P V T$ data for critical anomalies using pressure and density or volume as variables is still widespread; since the range of antisymmetry in these variables is so limited, bias is readily introduced into the exponents derived in this way.

Below the critical temperature, the situation is more involved.

The phase boundary, along which both $\Delta p$ and $\Delta \mu$ are zero, is symmetric around the "rectilinear diameter" $\bar{\rho}=\left(\rho_{L}+\rho_{G}\right) / 2$. Since $\bar{\rho} \neq \rho_{c}$, we choose the variable $\bar{\rho}$ rather than $\rho_{c}$ for temperatures below $T_{c}$. With this variable, neither $\Delta \mu$ nor $\Delta p$ is antisymmetric over an extended range, cf. figures 1 to 3 ; the $\mu, \rho$ representation is still the better of the two, and far superior to $p, v$ representation. Of course, even for temperatures only slightly below $T_{c}$, the entire range of antisymmetry observed above $T_{c} \quad( \pm 50 \%$ in density) is inside the two-phase region.

\subsection{Analyticity of $\mu\left(\rho_{c}, t\right)$}

If the chemical potential were fully antisymmetric, as it is in the lattice gas, then assuming it is regular everywhere except perhaps on the phase boundary, $\mu\left(\rho_{c}, t\right)$ has to be regular in $t$ everywhere on the critical isochore. This is so because, due to the antisymmetry, we can write

$$
\mu\left(\rho_{c}, t\right)=\frac{1}{2}[\mu(+\Delta \rho, t)+\mu(-\Delta \rho, t)],
$$

the quantities in brackets being chosen in the onephase region and therefore being regular at all $t$. Since the antisymmetry of $\Delta \mu$ is not complete in real gases, we cannot assume this regularity of $\mu\left(\rho_{c}, t\right)$ to hold strictly. We will examine the extent to which it is valid by considering the specific heat, which by thermodynamics can be written as

$$
\rho C_{v}=-\rho T\left(\partial^{2} \mu / \partial T^{2}\right)_{\rho}+T\left(\partial^{2} p / \partial T^{2}\right)_{\rho} .
$$

In the lattice gas and in the scaling law formulation, where $\mu\left(\rho_{c}, T\right)$ is regular, $T\left(\partial^{2} \mu / \partial T^{2}\right) \rho_{c}$ gives no anomalous contribution to $\boldsymbol{C}_{v}$ on the critical isochore, the divergence of $C_{v}$ being accounted for entirely by the divergence of $T\left(\partial^{2} p / \partial T^{2}\right)_{\rho_{c}}$, as discussed in section 2 . In real systems, however, an anomaly of $\left(\partial^{2} \mu / \partial T^{2}\right)_{\rho_{c}}$ may be expected [9]. In fact, even in the simple case of a Van der Waals fluid, 20 percent of the jump in $C_{v}$ at the critical point is caused by a jump in $T\left(\partial^{2} \mu / \partial T^{2}\right)_{\rho_{c}}$, as was shown by Barieau [10].

From eq (3.1) it follows that in the two-phase region where $\mu$ and $p$ are functions of $T$ only, $\rho C_{v}$ is linear in the density, with slope $-T\left(d^{2} \mu / d T^{2}\right)$ and intercept $+T\left(d^{2} p / d T^{2}\right)$. Alternatively, $C_{v}$ is linear in the volume with slope $T\left(d^{2} p / d T^{2}\right)$ and intercept $-T\left(d^{2} \mu / d T^{2}\right)$. Experimental evidence on the density dependence of $C_{v}$ in the two-phase region near $T_{c}$ is unfortunately scarce. The best source is formed by the $C_{v}$ data for $\mathrm{He}^{4}$ by Moldover [11]. Analyzing his raw data we find that $-T\left(d^{2} \mu / d T^{2}\right)$ is fairly constant and equal to $3.5 \pm 0.2$ in reduced units $p_{c} / \rho_{c} T_{c}$. The $C_{v}$ data for Ar by Voronel [12] and for steam by Amirchanov [13] show little or no dependence of $-T\left(d^{2} \mu / d T^{2}\right)$ on temperature and values of about 17.5 and 46 , respectively, for this quantity (fig. 4). Thus there is no experimental evidence for rapid change or divergence of $T\left(d^{2} \mu / d T^{2}\right)$ in the 

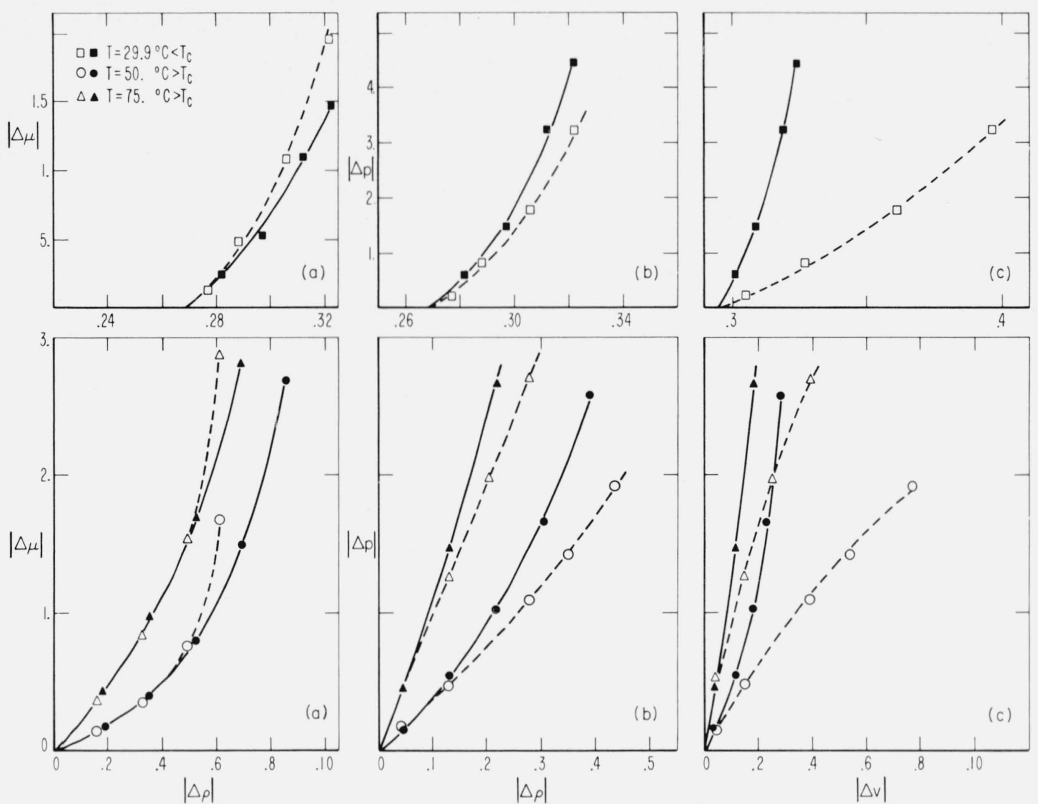

FigURE 1. The chemical potential difference $\Delta \mu$ as a function of the density difference $\Delta \rho(\mathrm{a})$, compared with the pressure difference $\Delta \mathrm{p}$ as a function of $\Delta \rho(\mathrm{b})$, and with $\Delta \mathrm{p}$ as a function of the volume difference $\Delta \mathrm{v}(\mathrm{c})$, for three isotherms of $\mathrm{CO}_{2}$ (ref. [6]).

Open symbols and dashed lines correspond to the low density side of the isotherms; closed symbols and solid lines to the high density side.
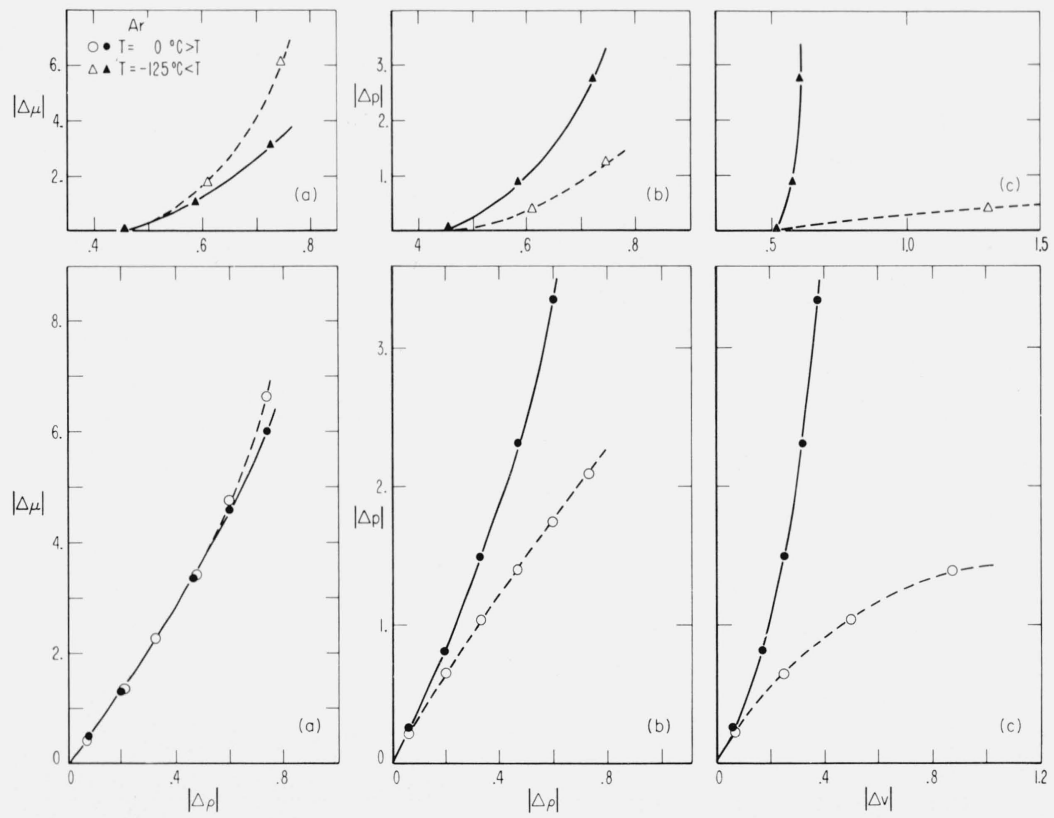

FIGURE 2. The chemical potential difference $\Delta \mu$ as a function of the density difference $\Delta \rho(\mathrm{a})$, compared with the pressure difference $\Delta \mathrm{p}$ as a function of $\Delta \rho(\mathrm{b})$, and with $\Delta \mathrm{p}$ as a function of the volume difference $\Delta \mathrm{v}(\mathrm{c})$, for two isotherms of $\operatorname{Ar}($ ref. [7]).

Open symbols and dashed lines correspond to the low density side of the isotherms; closed symbols and solid lines to the high density side. 

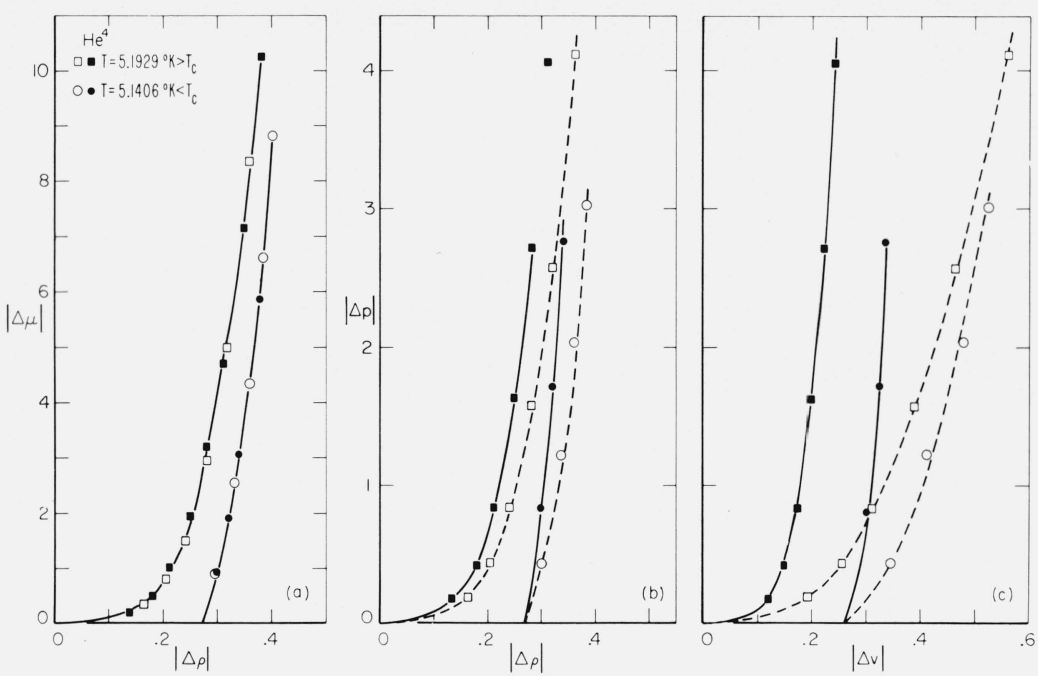

FIGURE 3. The chemical potential difference $\Delta \mu$ as a function of the density difference $\Delta \rho(\mathrm{a})$, compared with the pressure difference $\Delta \mathrm{p}$ as a function of $\Delta \rho(\mathrm{b})$, and with $\Delta \mathrm{p}$ as a function of the volume difference $\Delta \mathrm{v}(\mathrm{c})$, for two isotherms of $\operatorname{He}^{4}($ ref. $[8])$.

Open symbols and dashed lines correspond to the low density side of the isotherms; closed symbols and solid lines to the high density side.

two-phase region. In all these cases, on the contrary, rapid increase of $T\left(d^{2} p / d T^{2}\right)$ near $T_{c}$ is clearly indicated (fig. 4).

In the one-phase region, the quantities $\left(\partial^{2} p / \partial T^{2}\right)_{\rho}$ and $\left(\partial^{2} \mu / \partial T^{2}\right)_{\rho}$ in eq (3.1) are functions of density and temperature, thus this equation does not tell us anything about $T\left(\partial^{2} \mu / d T^{2}\right)_{\rho_{c}}$. The scaling laws, however, predict that also in the one-phase region there is only one contribution to $\rho C_{v}$ which is antisymmetric in the density (cf. eq (2.21)). This term is linear in $\rho$ and its coefficient is $-T\left(\partial^{2} \mu / \partial T^{2}\right)_{\rho_{c}}$. In the cases of $\mathrm{He}^{4}, \mathrm{Ar}$ and steam [13], this linearity of the antisymmetric part of $\rho C_{v}$ seems to hold rather well in the one-phase region, and its slope varies little with temperature and is close to the value observed in the two-phase region. Specifically, for $\mathrm{He}^{4}$ we find 3.5 for

$$
-T\left(\partial^{2} \mu / \partial T^{2}\right)_{\rho_{c}}
$$

above $T_{c}$ from the linear antisymmetric part of Moldover's supercritical $C_{v}$ data, and $3.9 \pm .4$ from Hill and Lounasmaa's $C_{v}$ data somewhat further away from critical [14]. We conclude that the scant experimental evidence presently available suggests that $\left(\partial^{2} p / \partial T^{2}\right)_{\rho_{c}}$ depends on temperature much more strongly than $\left(\partial^{2} \mu / \partial T^{2}\right)_{\rho_{c}}$. Thus, the hypothesis that $\mu\left(\rho_{c}, T\right)$ is regular at ${ }^{\mathrm{T}_{c}}$ is not contradicted by experimental evidence; if very careful measurements of the specific heat near the critical point would show an anomaly in $\left(\partial^{2} \mu / \partial T^{2}\right)_{\rho_{c}}$, then the basic hypothesis of the scaling laws, that both $\mu$ and $\Delta \mu$ are analytic throughout the one-phase region, would be invalid and the scaling in terms of $\Delta \mu, \Delta \rho, t$ would only be an approximation.

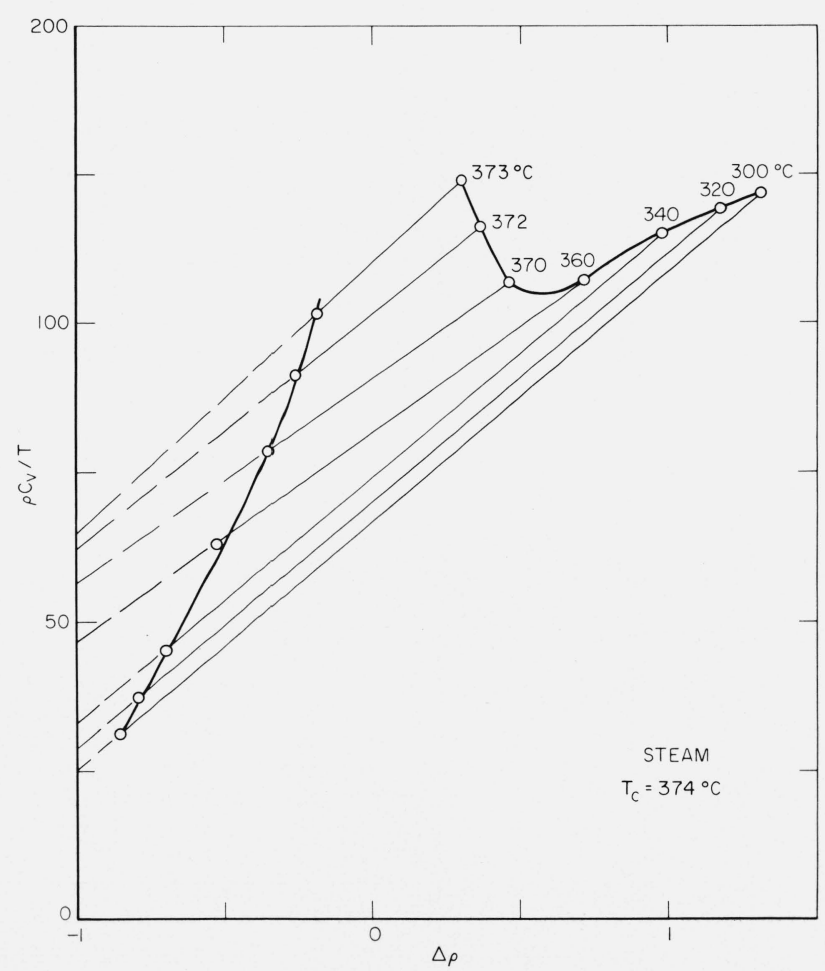

FIGURE 4A. $\quad\left(\rho \mathrm{C}_{\mathrm{v}} / \mathrm{T}\right)_{\text {II }}$ for steam (ref. [13]) is reduced units as a function of the reduced density differences $\Delta \rho$.

The slope of the specific heat isotherms is $\left(d^{2} \mu / d T^{2}\right)_{\text {II }}$ and does not show any appreciable variations. The intercept of the isotherms at $\Delta \rho=-1$ equals $\left(d^{2} p / d T^{2}\right)_{\mathrm{II}}$ and increases by over a factor 2 from 300 to $373^{\circ} \mathrm{C}$. 


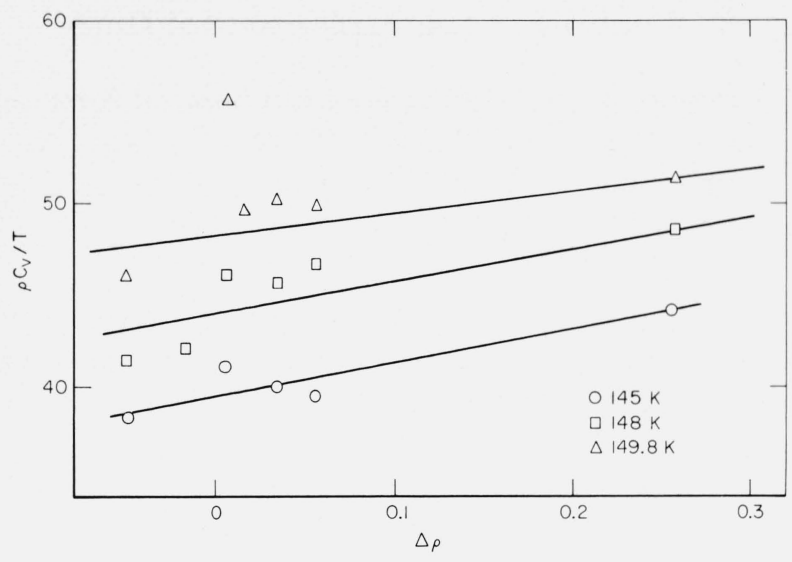

FigURE 4B. $\left(\rho \mathrm{C}_{\mathrm{v}} / \mathrm{T}\right)_{\mathrm{II}}$ for Argon (ref. [12]) in reduced units as a function of the reduced density difference $\Delta \rho$ in the two-phase region.

The slope of the specific heat isotherms is $-\left(d^{2} \mu / d T^{2}\right)_{\text {II }}$ and does not show appreciable variations. The intercept at $\Delta \rho=-1$ is $\left(d^{2} p / d T^{2}\right)_{\|}$and increases considerably.

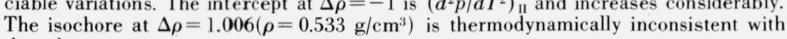
the others.

\section{A Preliminary Check of the Scaling Property}

The proposed equation of state, eq (2.2), implies an interesting scaling property which can be formulated in a variety of ways. For instance, if the chemical potential difference $|\Delta \mu|$ is divided by $|t|^{\beta \delta}$, eq (2.2) predicts that the result is a universal function of the variable $x$ only.

$$
|\Delta \mu| /|t|^{\beta \delta}=G(y)
$$

where

$$
y=|x|^{-\beta}=|\Delta \rho| /|t|^{\beta} \text {. }
$$

Using this representation we have shown [5] that this scaling property holds within certain bounds in the critical region of fluids. The scaled chemical potential differences of $\mathrm{CO}_{2}$ [6], $\mathrm{Xe}$ [15], $\mathrm{SF}_{6}$ [16], $\mathrm{N}_{2} \mathrm{O}$ [17], and $\mathrm{CClF}_{3}$ [17], obtained partially from classical PVT data, partially from optical density versus height measurements, fall on two branches of a universal curve. The representation (4.1) transforms the critical isotherm to infinity. This is a drawback which can be avoided by considering other representations. In the present paper we will instead scale $|\Delta \mu|$ by $|\Delta \rho|^{\delta}$, thus obtaining the universal function $h(x)$. With this representation the critical isotherm $(x=0)$ is simply a point inside the range of definition of $x$. In the previous analysis the scatter around the curve was appreciable for several reasons. First of all, one should expect deviations from corresponding states between these various substances. Secondly the choice of parameters $\beta$ and $\delta$ was not optimized and it was not investigated whether these exponents are indeed the same for all substances. Thirdly, it was not certain that for all the points correction terms to the asymptotic form of the equation of state at the critical point gave a negligible contribution. In the case of the van der Waals gas and in particular cases in the Ising model, the form of the correction terms and the range in which their contribu- tion is negligible are known; however, nothing is known a priori for real gases. Finally, the possibility of a systematic experimental error in part of the data analyzed in reference [5] is not excluded. In this paper we report the results of a detailed study of the experimental behavior of fluids in the critical region in which we have given attention to all these questions. First we have not assumed corresponding states but rather studied the gases substance by substance. This severely limits the choices of gases: of the substances analyzed in reference [5] only for $\mathrm{CO}_{2}$ and $\mathrm{Xe}$ the data are sufficient for a detailed analysis, to which we can add the recent data on $\mathrm{He}^{4}$ by Roach [8].

By proposing, furthermore, an explicit expression for $h(x)$ it was possible to transform the scaling problem into a linear least-squares problem. A goodness of fit criterion could be established and the scaling optimized by varying the parameters $\delta$ and $T_{c}$. Finally, for some of the substances, a tentative determination of the extension of the range of validity of the asymptotic form of the equation of state was made.

\section{Proposed Form for $\boldsymbol{h}(\boldsymbol{x})$}

The series expansion for $h(x)$ valid for large $x$, eq (2.6), contains terms of the form $x^{\gamma-2 \beta n}$. For real fluids the value of the exponents $\gamma$ and $\beta$ are close to $4 / 3$ and $1 / 3$ respectively. A closed form for $h(x)$, having all the required properties, will not be found in a simple real expression like the ratio of two polynomials [4]. The requirement that $h(x)$ be zero at $x=-x_{0}$, will be satisfied if, following the suggestion of Widom [2], we write

$$
h(x)=\frac{x+x_{0}}{x_{0}} \Phi(x) .
$$

The behavior of the experimental data, using reasonable values for $\beta$ and $\delta$, shows a linear dependence of $h(x)$ on $\left(x+x_{0}\right)$ in an appreciable range of $x$ including $x=0$, suggesting that $\Phi(x)$ may be considered as a correction term important for large values of $x$. Assume:

$$
\begin{aligned}
\Phi(x)=E_{1}\left[1+E_{2}\right. & \left.\left(\frac{x+x_{0}}{x_{0}}\right)^{2 \beta}\right]^{(\delta-1) / 2-1 / 2 \beta} \\
& =E_{1}\left[1+E_{2}\left(\frac{x+x_{0}}{x_{0}}\right)^{2 \beta}\right]^{(\gamma-1) / 2 \beta}
\end{aligned}
$$

with $E_{1}, E_{2}$ constants. The corresponding expression for $h(x)$ :

$h(x)=E_{1}\left(\frac{x+x_{0}}{x_{0}}\right)\left[1+E_{2}\left(\frac{x+x_{0}}{x_{0}}\right)^{2 \beta}\right]^{(\gamma-1) / 2 \beta}$

satisfies the following requirements:

(a) is analytical everywhere for $-x_{0}<x<\infty$,

(b) $\beta \delta h(x)>x h^{\prime}(x)$ : the isothermal compressibility is not negative,

(c) $h^{\prime \prime}(x) \geqslant 0$ : sufficient for the positivity of the heat capacity,

(d) at $x=-x_{0}, h(x)$ is nonanalytical but $h^{\prime}\left(-x_{0}\right)=E_{1}$ 
is finite: the compressibility on the phase boundary is finite and behaves like $(-t)^{-\gamma}$,

(e) on the critical isochore $(x \rightarrow \infty), h(x)$ has the series expansion:

$$
h(x)=x^{\gamma}\left[\sum_{n=0}^{\infty} a_{n} x^{-2 \beta n}+\sum_{n=1}^{\infty} b_{n} x^{-n}\right] .
$$

The first term of this series expansion leads to the correct behavior of the isothermal compressibility. The second series on the right-hand side of this equation, however, gives spurious terms. Since experimentally $2 \beta<1$ but $4 \beta>1$, the leading extra term $x^{\gamma-1}$ is then the third in the large $x$ expansion of $h(x)$.

The equivalent expansion of $\Delta \mu$ in terms of $\Delta \rho$ at constant $t>0$ is

$$
\Delta \mu=\Delta \rho\left[\sum_{n=0}^{\infty} f_{n}(t) \Delta \rho^{2 n}+\sum_{n=1}^{\infty} g_{n}(t)|\Delta \rho|^{n / \beta}\right] .
$$

The first two terms $f_{0}(t) \Delta \rho$ and $f_{1}(t) \Delta \rho^{3}$ are of the correct form but the third term $g_{1}(t)|\Delta \rho|^{1 / \beta} \cdot \Delta \rho$ will lead to a divergence of the fourth derivative of $\Delta \mu$ with respect to $\Delta \rho$ at $\Delta \rho=0$. Thus the function (5.3) fulfills most of the conditions imposed upon $h(x)$ but does not give the correct behavior for density derivatives of $\Delta \mu$ higher than the third on the critical isochore. However we do not consider this a serious drawback. Experimental $\Delta \mu$ data near the critical isochore are usually beset with so many uncertainties that determination of these higher derivatives is impossible anyway.

Expression (5.3) is a form for $h(x)$ with a small number of adjustable parameters, namely, $x_{0}, \beta, \delta, E_{1}$ and $E_{2}$, which is suitable for a linear least-squares analysis of the experimental data. Defining

$$
g(x)=\left[\frac{x_{0} h(x)}{x+x_{0}}\right]^{2 \beta /(\gamma-1)}=[\Phi(x)]^{2 \beta /(\gamma-1)}
$$

the assumption (5.2) for $\Phi(x)$ implies linear dependence of $g(x)$ on $\left[\left(x+x_{0}\right) / x_{0}\right]^{2 \beta}$ according to

$$
g(x)=E_{1}^{2 \beta /(\gamma-1)}\left[1+E_{2}\left(\frac{x+x_{0}}{x_{0}}\right)^{2 \beta}\right] .
$$

Explicit expression for the parameters $\Delta, \Gamma, \Gamma^{\prime}$ and the jump in specific heat across the phase boundary, in terms of the parameters which appear in the suggested form for $h(x)$, eq (5.3), can be derived from eq (2.4) to (2.8 and 2.25) and are as follows;

$$
\begin{aligned}
\Delta & =E_{1}\left[1+E_{2}\right]^{(\gamma-1) / 2 \beta} \\
\Gamma^{-1} & =x_{0}^{-\gamma} E_{1}\left[E_{2}\right]^{(\gamma-1) / 2 \beta} \\
\left(\Gamma^{\prime}\right)^{-1} & =(1 / \beta) x_{0}^{-\gamma} E_{1}, \Gamma / \Gamma^{\prime}=\beta\left[E_{2}\right]^{(1-\gamma) / 2 \beta} \\
\Delta\left(\rho C_{v}\right) & =\beta x_{0}^{\alpha-2} E_{1}(-t)^{-\alpha}
\end{aligned}
$$

The values of the parameters $A^{+}, A_{\overline{\mathrm{II}}}$ and $A_{\mathrm{I}}^{-}$can be evaluated numerically from (2.24) with the explicit form of $h(x)$. (See appendix II for details.)

\section{Analysis of the Experimental Data}

\subsection{Evaluation of the Difference in Chemical Potential $\Delta \mu$ from $\boldsymbol{p} v$ Isotherms}

The difference in chemical potential

$$
\Delta \mu=\mu(\rho, t)-\mu\left(\rho_{c}, t\right)
$$

along isotherms for $\mathrm{CO}_{2}$ [6], $\mathrm{Xe}$ [15], and $\mathrm{He}^{4}$ [8] is obtained by graphical integration of the pressure volume experimental isotherms according to

$$
\Delta \mu=\int_{\mu \underline{\left(\rho_{c}, t\right)}}^{\mu(\rho, t)} d \mu=\int_{p\left(\rho_{c}, t\right)}^{p(\rho, t)} v d p .
$$

In performing the integration the knowledge of the lower limits, the parameters $\rho_{c}$ and $p\left(\rho_{c}, t\right)$, is crucial. Experimentally, these parameters are known only to some degree of approximation and their uncertainty could introduce a systematic error in the analysis of the data. However, since $\Delta \mu(\rho, t)$ is with good accuracy an antisymmetric function of $\Delta \rho$ this property can be used as a criterion to establish the position of the point $\left[\rho_{c}, p\left(\rho_{c}, t\right)\right]$ along an isotherm. That is, we will define $\rho_{c}$ and $p\left(\rho_{c}, t\right)$ as the coordinates of the point with respect to which $\Delta \mu$ is an antisymmetric function of $\Delta \rho$ in an appreciable range of $\Delta \rho$. Of course the values of $\rho_{c}$ must be the same for all isotherms. At the same time, when the data extend over a large range of density, an estimate of the extension of the range of antisymmetry can be given. Actually this has been possible only for $\mathrm{CO}_{2}$ and $\mathrm{He}^{4}$; the range of antisymmetry extends to about 50 percent in density for $\mathrm{CO}_{2}$ and to about 35 percent for $\mathrm{He}^{4}$ for supercritical isotherms while it appears to be smaller in both cases for $T<T_{c}$ [for $T<T_{c} \Delta \mu=\mu(\rho, t)-\mu\left(\rho_{\text {coex }}, t\right)=\mu(\rho, t)$ $\left.-\mu\left(\rho_{c}, t\right)\right]$. These are rough estimates which obviously depend on the experimental error of the data. Since our aim is to inspect the validity of an asymptotic form of the equation-of-state $\Delta \mu(\Delta \rho, t)$ which is antisymmetric in $\Delta \rho$, we will restrict the analysis to points which satisfy this symmetry requirement within experimental error.

\subsection{Gravity Effect}

For all three substances the experimental measurements have been carried out in cells of finite height $h$. When the critical point is approached, there is a large density gradient in the cell; consequently, the average density will be different from the density at the level where the pressure is measured. To correct for this error, an a priori knowledge of the density profile, or equivalently, of the chemical potential as a function of density, would be needed. Since this is the quantity to be determined in the first place, this knowledge is not available. Thus the option left is to omit those data that are greatly beset with this error. The effect is most pronounced very near the critical point, and, in terms of pressure, the correction is at most $\rho_{c} g h$. If the difference between the measured pressure and the 
TABLE 1. $\mathrm{CO}_{2}$, chemical potential difference $\Delta \mu=\mu(\rho, \mathrm{t})-\mu\left(\rho_{\mathrm{c}}, \mathrm{t}\right)$ at various densities $\Delta \rho=\left(\rho-\rho_{\mathrm{c}}\right) / \rho_{\mathrm{c}}$ and temperatures $\mathrm{T}$ for $\mathrm{CO}{ }_{2}$

The $\Delta \rho, \Delta \mu$ data are in reduced units, the factors used for reducing the experimental data of ref. [6] are $\rho_{c}=236.7$ Amagat and $p_{c} / \rho_{c}=0.3075$ atm Am. ${ }^{-1}$

\begin{tabular}{|c|c|c|c|c|c|c|c|c|}
\hline$T^{\circ} \mathrm{C}$ & $10^{2} \Delta \rho$ & $10^{4} \Delta \mu$ & $T^{\circ} \mathrm{C}$ & $10^{2} \Delta \rho$ & $10^{4} \Delta \mu$ & $T^{\circ} \mathrm{C}$ & $10^{2} \Delta \rho$ & $10^{4} \Delta \mu$ \\
\hline 29.929 & 28.34 & 8.2 & 31.185 & $\begin{array}{r}4.76 \\
9.48 \\
15.03 \\
20.93 \\
-3.73 \\
-6.99 \\
-10.57 \\
-15.18 \\
-21.00\end{array}$ & $\begin{array}{r}1.3 \\
3.5 \\
10.8 \\
31.0 \\
-0.8 \\
-2.4 \\
-4.2 \\
-10.4 \\
-30.9\end{array}$ & 32.054 & $\begin{array}{r}3.86 \\
13.98 \\
22.32 \\
-5.11 \\
-11.94 \\
-19.05 \\
-24.65 \\
-29.21\end{array}$ & $\begin{array}{r}6.2 \\
34.1 \\
87.5 \\
-8.8 \\
-24.0 \\
-59.2 \\
-112.2 \\
-185.0\end{array}$ \\
\hline 30.409 & $\begin{array}{r}23.82 \\
-24.22\end{array}$ & $\begin{array}{r}6.0 \\
-7.7\end{array}$ & 31.320 & $\begin{array}{r}4.72 \\
9.61 \\
15.13 \\
20.96 \\
-3.72 \\
-6.93 \\
-10.58 \\
-15.23 \\
-20.97\end{array}$ & $\begin{array}{r}1.7 \\
5.3 \\
15.0 \\
37.7 \\
-1.7 \\
-3.6 \\
-6.7 \\
-15.1 \\
-37.7\end{array}$ & 34.720 & $\begin{array}{r}9.76 \\
21.41 \\
-10.96 \\
-19.95 \\
-27.37\end{array}$ & $\begin{array}{r}81.6 \\
235.1 \\
94.0 \\
-207.2 \\
-364.2\end{array}$ \\
\hline 31.013 & $\begin{array}{r}13.02 \\
15.20 \\
21.21 \\
27.14 \\
-12.88 \\
-15.50 \\
-18.59 \\
-21.79\end{array}$ & $\begin{array}{r}3.1 \\
6.1 \\
24.2 \\
70.6 \\
-2.8 \\
-6.1 \\
-13.9 \\
-27.3\end{array}$ & 31.523 & $\begin{array}{r}9.50 \\
14.67 \\
20.28 \\
-8.60 \\
-15.02 \\
-21.17 \\
-26.45\end{array}$ & $\begin{array}{r}8.3 \\
19.1 \\
42.9 \\
-7.6 \\
-20.1 \\
-49.4 \\
-101.5\end{array}$ & 40.087 & $\begin{array}{r}.95 \\
11.34 \\
-6.98\end{array}$ & $\begin{array}{r}24.6 \\
276.1 \\
-157.4\end{array}$ \\
\hline
\end{tabular}

pressure on the critical isochore is less than three times this amount, we have rejected the point.

\section{The Data}

A critical examination of the internal consistency of each set of data has been performed before proceeding to the analysis. This led to the following conclusions.

(a) $\mathrm{CO}_{2}$ data [6]. The internal consistency is good. Since the cell height was unspecified and varied from point to point, the gravity cutoff was arbitrarily taken at $\Delta p=0.006$ bar. The data for $\Delta \mu, \Delta \rho, T$ are listed in table 1 .

(b) Xe data [15]. The cell height being $1 \mathrm{~cm}$, the gravity cutoff is at 0.003 bar. The data have been taken along isotherms and along isochores. As shown in figure 5 , there is a discrepancy between the two sets of measurements. The reason for the discrepancy is not clear; however in the isochoric measurements check points taken at the beginning and at the end of each run are in agreement, while in the isothermal measurements no check points are available. We will therefore assume that the isochoric measurements are more reliable. We will then analyze the $\Delta \mu, \Delta \rho$ data evaluated from isotherms constructed from the isochoric data. This leaves us with a relatively small amount of data and therefore the result of the analysis will be of a somewhat qualitative character. The data are listed in table 2.
TABLE 2. Xe, chemical potential difference $\Delta \mu=\mu(\rho, \mathrm{t})-\mu\left(\rho_{\mathrm{c}}, \mathrm{t}\right)$ at various densities $\Delta \rho=\left(\rho-\rho_{\mathrm{c}}\right) / \rho_{\mathrm{c}}$ and temperatures $\mathrm{T}$ for $\mathrm{Xe}$

The $\Delta \rho, \Delta \mu$ data are in reduced units, the factors used for reducing the experimental data of ref. [5] are $\rho_{c}=1.110 \mathrm{~g} / \mathrm{cm}^{3}$ and $p_{c} / \rho_{c}=51.84\left(\mathrm{~cm}^{3} / \mathrm{g}\right) \mathrm{atm}$.

\begin{tabular}{|c|c|c|c|c|c|}
\hline$T^{\circ} \mathrm{C}$ & $10^{2} \Delta \rho$ & $10^{4} \Delta \mu$ & $T^{\circ} \mathrm{C}$ & $10^{2} \Delta \rho$ & $10^{4} \Delta \mu$ \\
\hline 16.790 & $\begin{array}{r}3.01 \\
6.14 \\
14.57 \\
-4.99 \\
-9.16 \\
-13.19 \\
-13.47\end{array}$ & $\begin{array}{r}0.5 \\
1.5 \\
9.5 \\
-1.0 \\
-3.7 \\
-7.4 \\
-8.3\end{array}$ & 17.590 & $\begin{array}{r}6.14 \\
14.57 \\
-0.95 \\
-2.51 \\
-3.87 \\
-4.99 \\
-9.16 \\
-13.19\end{array}$ & $\begin{array}{r}9.5 \\
31.5 \\
-1.4 \\
-3.5 \\
-5.7 \\
-7.1 \\
-15.8 \\
-26.9\end{array}$ \\
\hline 16.990 & $\begin{array}{r}3.01 \\
6.14 \\
14.57 \\
-4.99 \\
-9.16 \\
-13.19 \\
-13.47\end{array}$ & $\begin{array}{r}1.5 \\
3.6 \\
15.5 \\
-1.9 \\
-6.1 \\
-11.8 \\
-13.1\end{array}$ & 17.990 & $\begin{array}{r}6.14 \\
14.57 \\
-0.95 \\
-2.51 \\
-2.79 \\
-3.87 \\
-4.99 \\
-9.16 \\
-13.19\end{array}$ & $\begin{array}{r}14.1 \\
43.2 \\
-2.2 \\
-5.3 \\
-5.4 \\
-8.3 \\
-10.8 \\
-23.1 \\
-37.8\end{array}$ \\
\hline 17.190 & $\begin{array}{r}6.14 \\
14.57 \\
-4.99 \\
-9.16 \\
-13.19 \\
-13.47\end{array}$ & $\begin{array}{r}5.8 \\
20.6 \\
-2.7 \\
-8.6 \\
-16.1 \\
-17.7\end{array}$ & 18.390 & $\begin{array}{r}3.01 \\
6.14 \\
-0.95 \\
-3.87 \\
-4.99 \\
-9.16 \\
-13.19 \\
-13.47\end{array}$ & $\begin{array}{r}9.0 \\
19.3 \\
-2.7 \\
-11.5 \\
-14.5 \\
-29.6 \\
-48.6 \\
-50.5\end{array}$ \\
\hline
\end{tabular}




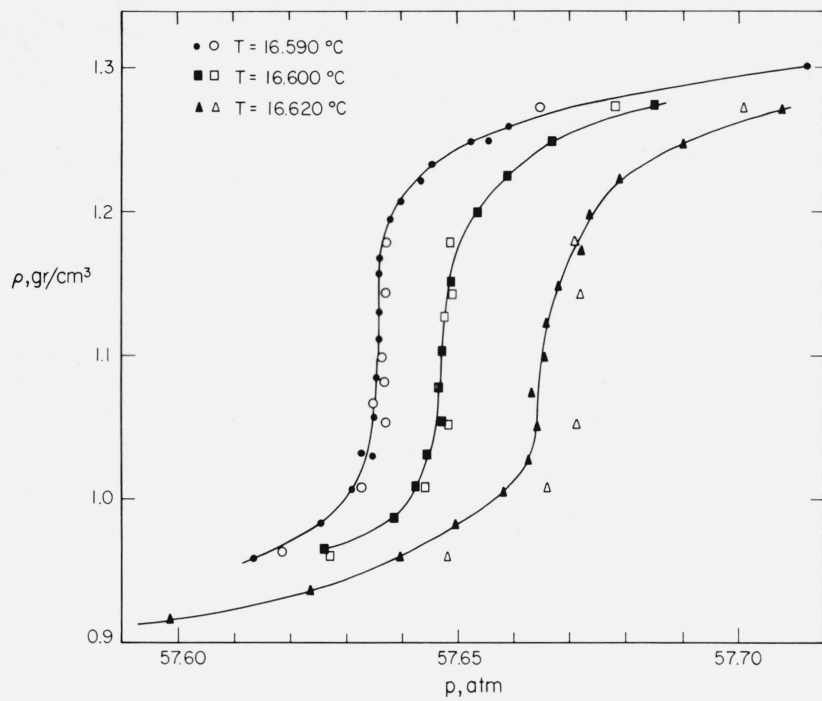

Figure 5. Comparison between isothermal and isochoric measurements on $\mathrm{Xe}$ [15].

Full symbols and the solid lines show the isothermal measurements. Open svmbols show the position of points obtained in isochoric measurements; for the data at $T=16.590^{\circ} \mathrm{C}$ these are direct experimental data, for the other isotherms they are obtained by linear these are direct experimental data, for the other isoth
interpolation of the $p, t$ isochores in a range of $0.01{ }^{\circ} \mathrm{C}$.

(c) $\mathrm{He}^{4}[8]$. In this experiment the dielectric constant of a very thin layer of $\mathrm{He}^{4}(0.025 \mathrm{~cm})$ between two condenser plates was measured as a function of temperature and pressure. There are three sources of systematic error to be discussed. The first is the gravity effect. Although density gradients between the condenser plates are negligible, the pressure was measured at a level above the cryostat. Even if the head correction to the top of the cell could be accurately estimated, an unknown head approximately $2.5 \mathrm{~cm}$ above the level of the condenser from the top of the cell, is present. This gives a gravity cutoff of 0.0003 bar. The second source of error lies in the conversion of dielectric constant to density using a Clausius-Mosotti relation with constant molar polarizability $\alpha=0.1230 \mathrm{~cm}^{3} / \mathrm{mol}$. However, if $\alpha$ depends on the density as observed for $\mathrm{He}^{3}$ [18],

$$
\alpha\left(\mathrm{cm}^{3} / \mathrm{mol}\right)=0.1234-1.11 \times 10^{-4} \rho / \rho_{c},
$$

then the first order correction to a density difference $\Delta \rho$ calculated assuming constant $\alpha$ would be $1.11 \times 10^{-4}(1 / \alpha+\Delta \rho) \Delta \rho$. This correction is different at the low- and at the high-density side, and would, at $\Delta \rho \sim 30$ percent, amount to $2.6 \times 10^{-4}$ in $\Delta \rho$ on the vapor side and $2.8 \times 10^{-4}$ on the liquid side, respectively. Since the uncertainty in $\Delta \rho$ is about $3 \times 10^{-4}$, this is a borderline correction; however, if applied, it would slightly improve the antisymmetry of the $\Delta \mu, \Delta \rho$ data.

The third source of error is more elusive and is related to the use of the $\mathrm{T}_{58} \mathrm{He}^{4}$ vapor pressure scale [19] near the critical point of $\mathrm{He}^{4}$. This scale relates the vapor pressure to the temperature by an equation which is analytic at the critical point. This is contrary to the current ideas about critical point anomalies.
According to the scaling laws, the divergence of the specific heat imples a similar divergence in $d^{2} p_{v} / d T^{2}$. Moldover [11] estimates from his specific heat measurements that vapor pressures near the critical have to be assigned temperatures up to $0.0007 \mathrm{~K}$ below those on $\mathrm{T}_{58}$. One can avoid this difficulty by calibrating the thermometer independent of the $\mathrm{T}_{58}$ scale, or by calibrating it on this scale in a temperature range not too close to $T_{c}$ (say below $5 \mathrm{~K}$ ).

In the present experiment it appears that a germanium thermometer has been calibrated on the $T_{58}$ scale in the range from $5 \mathrm{~K}$ to the critical point, and, moreover, that this calibration was extrapolated to temperatures above critical. From Moldover's estimate of the size of the correction below $T_{c}$, and assuming that all temperatures are measured relative to critical, it seems corrections to $T_{c}-T$ will be below $0.5 \mathrm{mK}$. For $T>T_{c}$, where extrapolation is involved, the correction may well increase; we will assume that for isotherms further from critical the temperature is not known to better than $1 \mathrm{mK}$.

The $\Delta \mu, \Delta \rho, T$ data for $\mathrm{He}^{4}$ are listed in table 3. Other data on $\mathrm{He}^{4}$ are available in the work of Edwards [20]. These data are restricted to the region $T<T_{c}$ and yield information on the phase boundary and the behavior of the compressibility for $t<0$. We have chosen to check the scaling properties on Roach's data because in this case also the region above the critical temperature has been explored. We will compare the results with the results of Edwards only qualitatively because a direct comparison of the coexistence curves in the $\rho T$ plane by the two authors shows some as yet unresolved discrepancies.

\section{The Analysis of the $\Delta \mu, \Delta \rho, t$ Data}

Using conventional linear least-squares techniques, we fitted $g(x)$, defined by eq (5.8), as a function of $\left[\left(x+x_{0}\right) / x_{0}\right]^{2 \beta}$ and obtained estimates for $E_{1}, E_{2}$ and their errors. The computations were performed using the general purpose program OMNITAB [21]. The experimental values of $\Delta \mu, \Delta \rho$, and $T$ (tables 1-3) were used to evaluate $x$ and $g(x)$ by using suitable values for $\rho_{c}, \beta, \delta, x_{0}$, and $T_{c}$. We took $\beta$ and $x_{0}$ from a preliminary analysis of coexistence curves. A more refined analysis, to be published [22], yields values for $\beta$ and $x_{0}$ which are only slightly different from the ones we used (see table 4). The fit of the $\Delta \mu, \Delta \rho$, t data is repeated for different values of $\delta$ and $T_{c}$ until a minimum in the standard deviation of the fit is reached. The value for $T_{c}$ corresponding to the minimum agrees with the critical temperature obtained from fitting the coexistence curve [22] (table 4).

Weights were assigned to $\Delta \mu, \Delta \rho$, and $t$ in the following way. The precision of the experimental pressure, temperature and density was estimated from the description of the respective experimental methods. From the pressure error $\sigma_{p}$, the error in $\Delta \mu$ was estimated as $\sigma_{\mu}=2 \sigma_{p} / p_{c}$. The estimated errors in the reduced quantities $\Delta \mu, \Delta \rho, t$ are listed in table 5 . The fit was performed assuming that the variable $\left[\left(x+x_{0}\right) / x_{0}\right]^{2 \beta}$ is free of error and that the variable $g(x)$ 
TABLE 3. $\mathrm{He}^{4}$, chemical potential difference $\Delta \mu=\mu(\rho, \mathrm{t})-\mu\left(\rho_{\mathrm{c}}, \mathrm{t}\right)$ at various densities $\Delta \rho=\left(\rho-\rho_{\mathrm{c}}\right) / \rho_{\mathrm{c}}$ and temperatures $\mathrm{T}$ for $\mathrm{He} \mathrm{e}^{4}$ The $\Delta \rho, \Delta \mu$ data are in reduced units, the factors used for reducing the experimental data of ref. [8] are $\rho_{c}=0.069 \mathrm{~g} / \mathrm{cm}^{3}$ and $p_{c} / \rho_{c}=2.475 \mathrm{l} 10^{4}$ torr $\mathrm{cm} 3 / \mathrm{g}$.

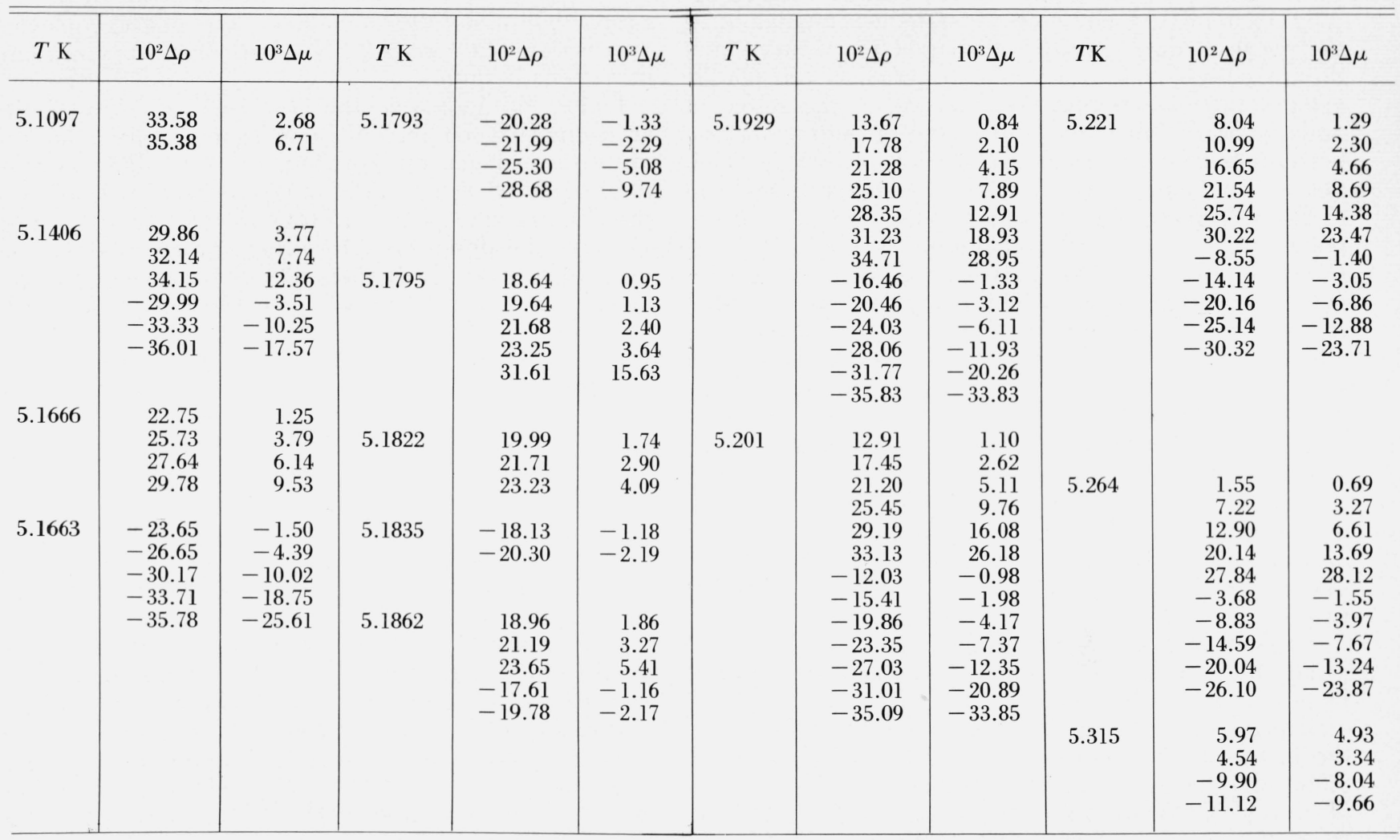

TABLE 4. Critical parameters for $\mathrm{CO}_{2}, \mathrm{Xe}$, and $\mathrm{He}^{4}$.

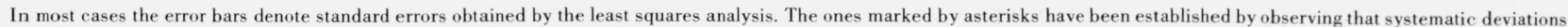
from the fitted equation are present if the parameter is varied by this amount or more.

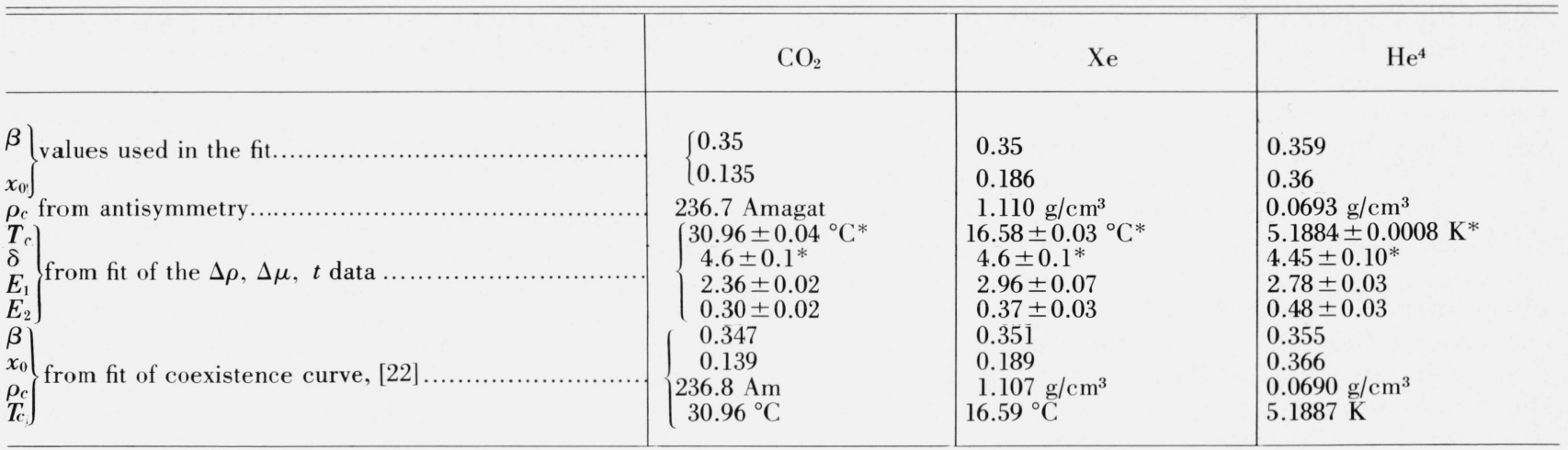

contains all experimental uncertainties $\sigma_{\mu}, \sigma_{\rho}, \sigma_{t}$. Using propagation of errors the weight to be attributed to $g(x)$ is found to be

$$
\begin{aligned}
W= & {\left[\frac{\gamma-1}{2 \beta}\right]^{2}\left\{\left(\frac{\sigma_{\mu}}{\Delta \mu}\right)^{2}+\left(\frac{\sigma_{\rho}}{\Delta \rho}\right)^{2}\left[\delta-\frac{x}{\beta\left(x+x_{0}\right)}\right]^{2}\right.} \\
& \left.+\left(\frac{\sigma_{t}}{t}\right)^{2}\left(\frac{x}{x+x_{0}}\right)^{2}\right\}^{-1}[g(x)]^{-2} .
\end{aligned}
$$

In order to obtain reliable values for the critical expo-
TABLE 5. Estimated experimental errors in the reduced density difference $\left(\sigma_{\rho}\right)$, reduced chemical potential difference $\left(\sigma_{\mu}\right)$ and in the reduced temperature $\left(\sigma_{t}\right)$

\begin{tabular}{c|c|c|c}
\hline \hline & $\sigma_{\rho}$ & $\sigma_{\mu}$ & $\sigma_{t}$ \\
\hline $\mathrm{CO}_{2} \ldots \ldots \ldots \ldots \ldots \ldots \ldots \ldots \ldots \ldots \ldots$ & $3.3 \times 10^{-4}$ & $6.5 \times 10^{-5}$ & $2.0 \times 10^{-5}$ \\
$\mathrm{Xe} \ldots \ldots \ldots \ldots \ldots \ldots \ldots$ & $2.0 \times 10^{-4}$ & $3.5 \times 10^{-5}$ & $3.4 \times 10^{-6}$ \\
$\mathrm{He} \ldots \ldots \ldots \ldots \ldots \ldots \ldots$ & $3.3 \times 10^{-4}$ & $1.2 \times 10^{-4}$ & $5.0 \times 10^{-5}$ \\
\hline
\end{tabular}


nents and coefficients, we have to make sure that contributions from possible corrections to the asymptotic form of the equation of state are negligible in the range of data considered. Correction terms which do not possess the antisymmetry property are readily excluded by restricting the analysis to the range of antisymmetry. Because the form of antisymmetric correction terms is unknown, their contributions cannot be evaluated. However, such terms will scale in a way different from the asymptotic term; therefore, points for which their contribution is important will show systematic deviations from the fit. For one substance, namely $\mathrm{CO}_{2}$, for which many data are available on isotherms substantially higher than critical, the effect of points outside the range of validity on the values of the parameters may be observed and an estimate of the range derived.

Actually, for $\mathrm{CO}_{2}$, omitting points within the range of antisymmetry for the isotherms between 29.929 and $34.720{ }^{\circ} \mathrm{C}$ does not affect the results of the fit while including points with $\Delta \rho>11$ percent at $40{ }^{\circ} \mathrm{C}$ and $\Delta \rho>1.4$ percent at $50^{\circ} \mathrm{C}$ (next point at $\left.15 \%\right)$ introduces systematic deviations and an apparent lowering of the value of $\delta$.

For Xe the temperature range of the data is quite small and no appreciable effect was observed omitting points at large densities on any isotherms. For $\mathrm{He}^{4}$ omitting points within the range of antisymmetry for the isotherms between $5.109 \mathrm{~K}$ and $5.202 \mathrm{~K}$ does not affect the results while the inclusion in the analysis of the points with $\Delta \rho>30$ percent at $T=5.221 \mathrm{~K}$, $\Delta \rho>28$ percent at $T=5.264 \mathrm{~K}$ and with $\Delta \rho>11$ percent at $T=5.313 \mathrm{~K}$ introduces systematic deviations which again apparently lower the value of $\delta$.

In table 4 , the values of the parameters obtained by fitting the $\Delta \mu, \Delta \rho, t$ data are listed. The value of $\rho_{c}$ is that with respect to which $\Delta \mu$ is antisymmetric. The parameters $E_{1}, E_{2}$ are least-squares estimates, as explained before; the errors listed are standard deviations. The parameters $\delta$ and $T_{c}$ were varied stepwise. The values given minimize the standard deviation of the fit. The errors in $\delta$ and $T_{c}$ are estimates obtained by observing that systematic deviations from the fitted equation arise if the parameters are varied by this amount or more. They do not take into account the effect of small variations in $\beta$ and $x_{0}$. For the three substances, the values found for the exponents $\beta$ and $\delta$ are the same within the error bounds. To decide if the small observed differences are significant, more accurate determinations of the exponents are needed. Of the other parameters describing the critical point behavior, $E_{1}$ and $E_{2}$ do not show large variations either; the only parameter varying greatly from substance to substance is $x_{0}$.

In figures $6 \mathrm{~A}$ to $8 \mathrm{~A}$ the optimized function $h(x)$ is compared with the experimental data for the three substances in a log log plot. It is seen that the $\log h(x)$ is a smooth and nearly linear function of $\log \left(x+x_{0}\right)$. Deviations from linearity are important only for quite large value of $x$. The point at $x=0$ (critical isotherm) behaves like any other point at finite $x$. The agreement with the experimental points is best seen from the deviations plots shown in figures $6 \mathrm{~B}$ to $8 \mathrm{~B}$ where the relative difference between the experimental and the calculated value of $h(x)$ is plotted versus $\log \left[\left(x+x_{0}\right) / x_{0}\right]$. No evidence of systematic deviations is found.

Figure $8 \mathrm{~B}$ indicates that for $\mathrm{He}^{4}$ the error limits are somewhat too narrow. Some of the errors in table 5 must have been underestimated. An adjustment of the estimated error in the pressure could remedy this problem. In view of the arbitrariness of this procedure, we have not done this. Moreover, statistical estimates for parameters and their standard deviations are usually not very sensitive to the exact weighing procedure used.

From the values of $\beta, \delta, E_{1}$ and $E_{2}$, and using the equalities

$$
\begin{gathered}
y=\beta(\delta-1) \\
2-\alpha=\beta(\delta+1)
\end{gathered}
$$

and definitions of $\Delta, \Gamma, \Gamma^{\prime} \ldots$., eq (5.9), we have determined the parameters listed in table 6 . If $\beta$ and $\delta$ are constant from substance to substance, obviously the same would be true for the exponents $\gamma$ and $\alpha$. Of the other parameters also $\Delta$ and the ratio $\Gamma / \Gamma^{\prime}$ seem to be remarkably steady. Therefore the critical isotherms of the three gases should approximately coincide; our previous study, implicitly assuming this coincidence, yielded a value of $\delta$ slightly higher than the one found presently. This must be due to the inclusion in the previous analysis of other data not studied here. The compressibility ratio $\Gamma / \Gamma^{\prime}$ is substantially smaller than our previous estimate obtained by graphical determination of slopes of isotherm (see below). Our new value agrees reasonably well with the value obtained for Xe by Wilcox and Balzarini [23] using optical interferometry, namely $\Gamma / \Gamma^{\prime}=4.9$.

For each substance, internal consistency checks have been made by comparing experimental critical isotherm and compressibility data with values predicted by our equation.

An example is figure 9 for $\mathrm{CO}_{2}$, where the inverse compressibility $(9 \mathrm{~A})$ on the critical isochore above $T_{c}$ and along the phase boundary below $T_{c}$ is shown; moreover, the critical isotherm is plotted (9B). For the latter, it is seen that the experimental data agree quite well with the calculated curve. This is also the case for the compressibility above $T_{c}$. However, below $T_{c}$, the experimental points show systematically lower compressibilities than our equation predicts. These points have been obtained by graphical determination of the slope of isotherms near the phase boundary, and by assuming linearity of $p$ versus $\Delta \rho$ in an appreciable range. The optical measurements [17] indicate strong curvature of these isotherms, suggesting that slopes taken from $P V T$ data tend to underestimate the limiting compressibility. The function (5.3) predicts a very small range of linearity; the discrepancy is then explained by the fact that the experimental points do not define the true limiting behavior. The refractive index measurements of Edwards [20] in $\mathrm{He}^{4}$ permit 


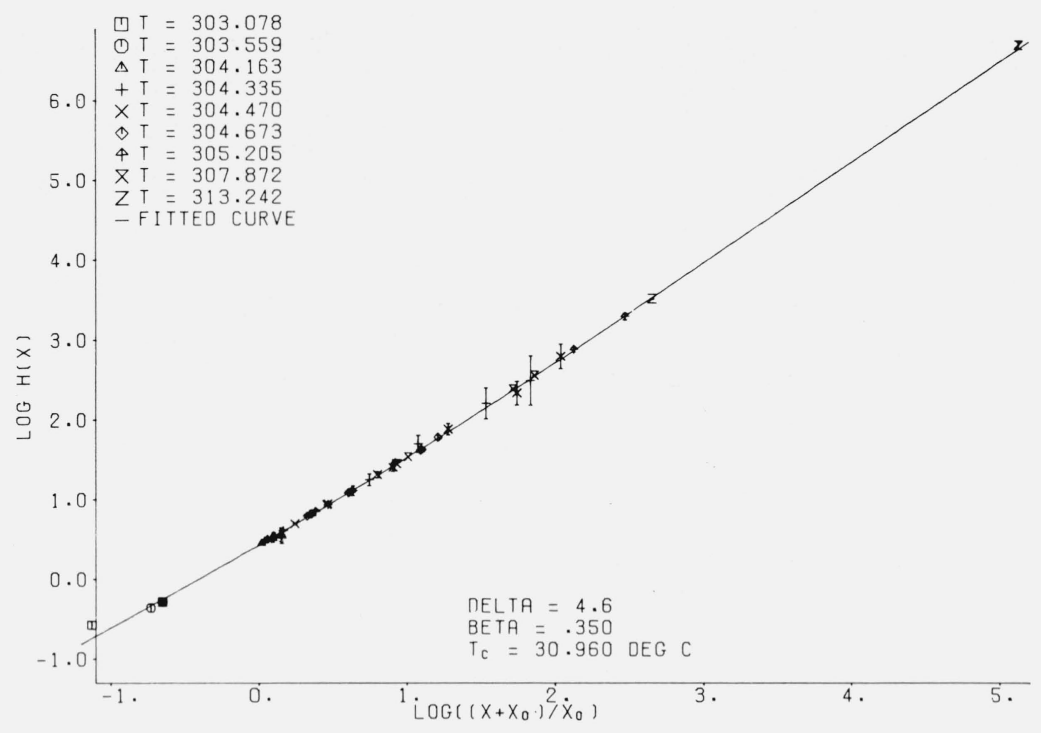

FIGURE 6A. Log-log plot of $\mathrm{h}(\mathrm{x})$ versus $\left(\mathrm{x}+\mathrm{x}_{0}\right) / \mathrm{x}_{0}$ for $\mathrm{CO}_{2}$.

The solid line represents $h(x)$ according to eq (5.3) with parameters given in table 4. Experimental points are shown with estimated errors in the variable $h(x)$.

RELATIVE DEVIATIONS: CO2

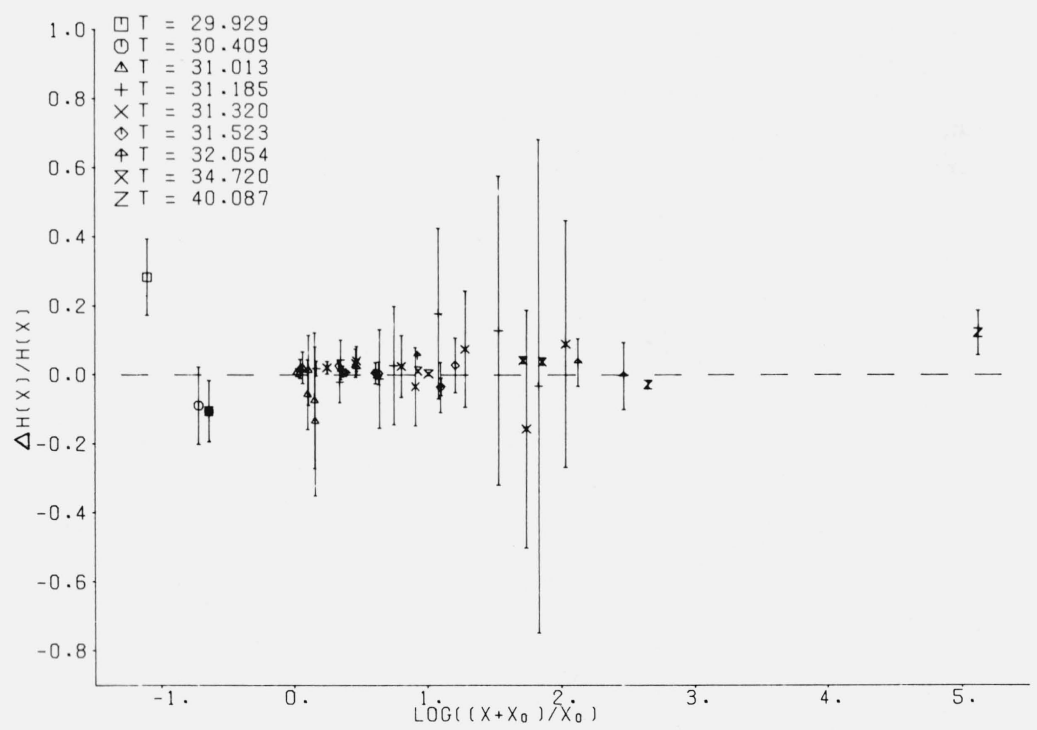

FIGURE 6B. Relative deviation $\Delta \mathrm{h}(\mathrm{x}) / \mathrm{h}(\mathrm{x})=\left[\mathrm{h}_{\text {exp }}(\mathrm{x})-\mathrm{h}_{\text {fitted }}(\mathrm{x})\right] / \mathrm{h}_{\text {exp }}(\mathrm{x})$ versus $\log \left[\left(\mathrm{x}+\mathrm{x}_{0}\right) / \mathrm{x}_{0}\right]$ for $\mathrm{CO}_{2}$.

Vertical error bars represent all experimental uncertainties. 


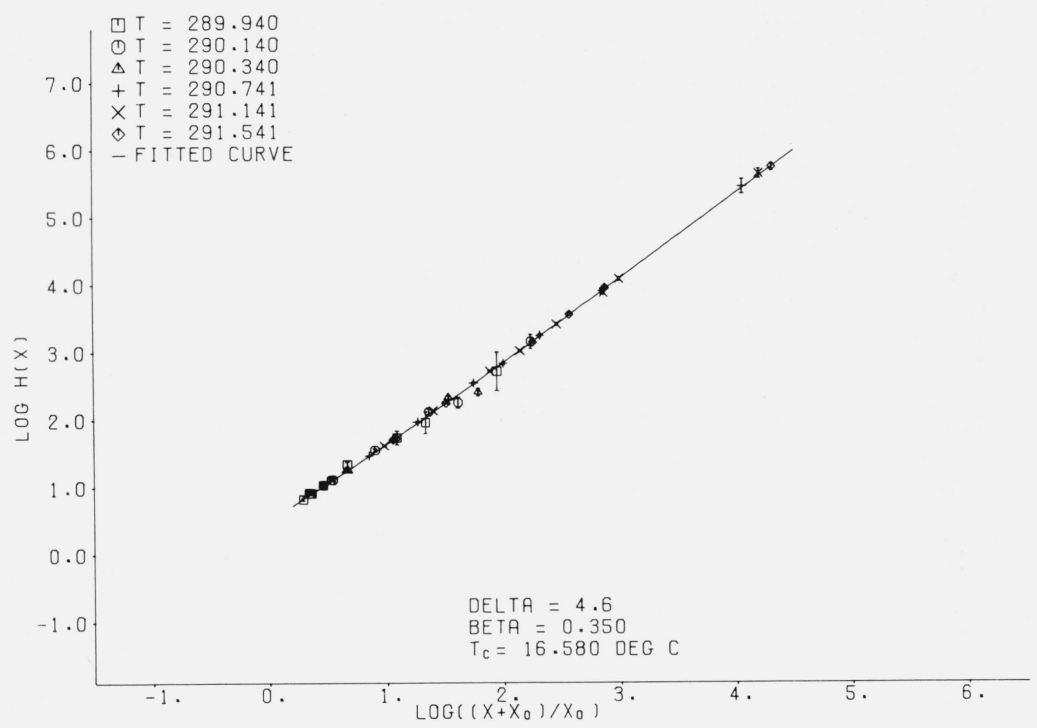

FigURE 7A. Log-log plot of $\mathrm{h}(\mathrm{x})$ versus $\left(\mathrm{x}+\mathrm{x}_{0}\right) / \mathrm{x}_{0}$ for $\mathrm{Xe}$.

The solid line represents $h(x)$ according to eq (5.3) with parameters given in table 4 . Experiment points are shown with estimated errors in the variable $h(x)$.

RELATIVE DEVIATIONS: XENON

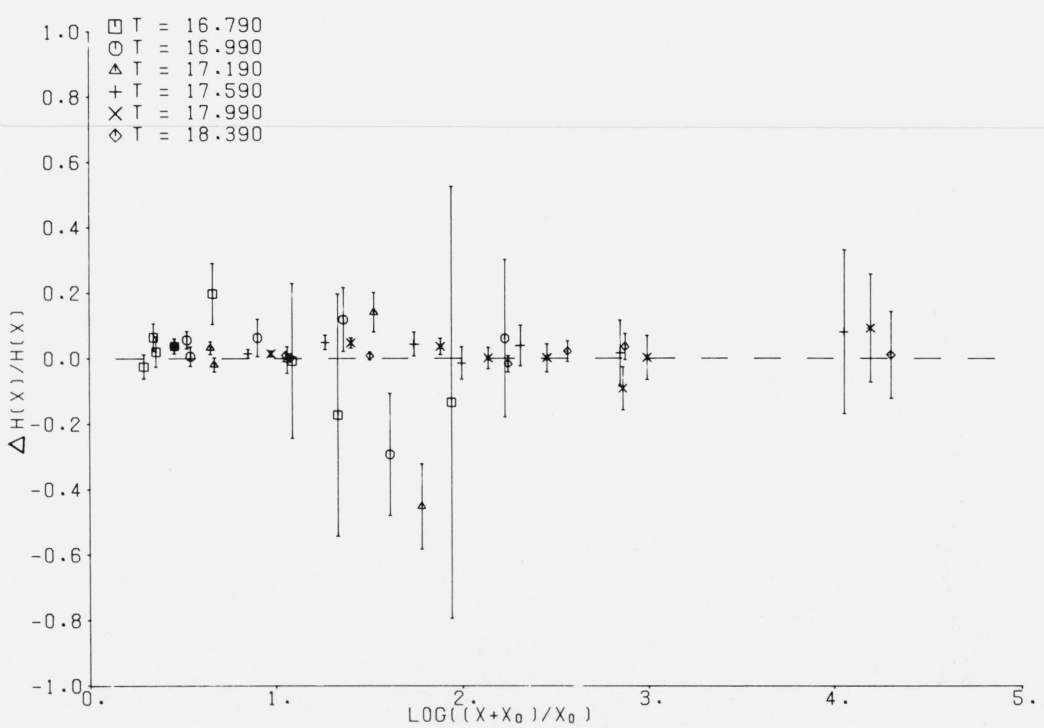

FigURE 7B. Relative deviation $\Delta \mathrm{h}(\mathrm{x}) / \mathrm{h}(\mathrm{x})=\left[\mathrm{h}_{\exp }(\mathrm{x})-\mathrm{h}_{\text {fitted }}(\mathrm{x})\right] / \mathrm{h}_{\exp }(\mathrm{x})$ versus $\log \left[\left(\mathrm{x}+\mathrm{x}_{0}\right) / \mathrm{x}_{0}\right]$ for $\mathrm{Xe}$.

Vertical error bars represent all experimental uncertainties. 


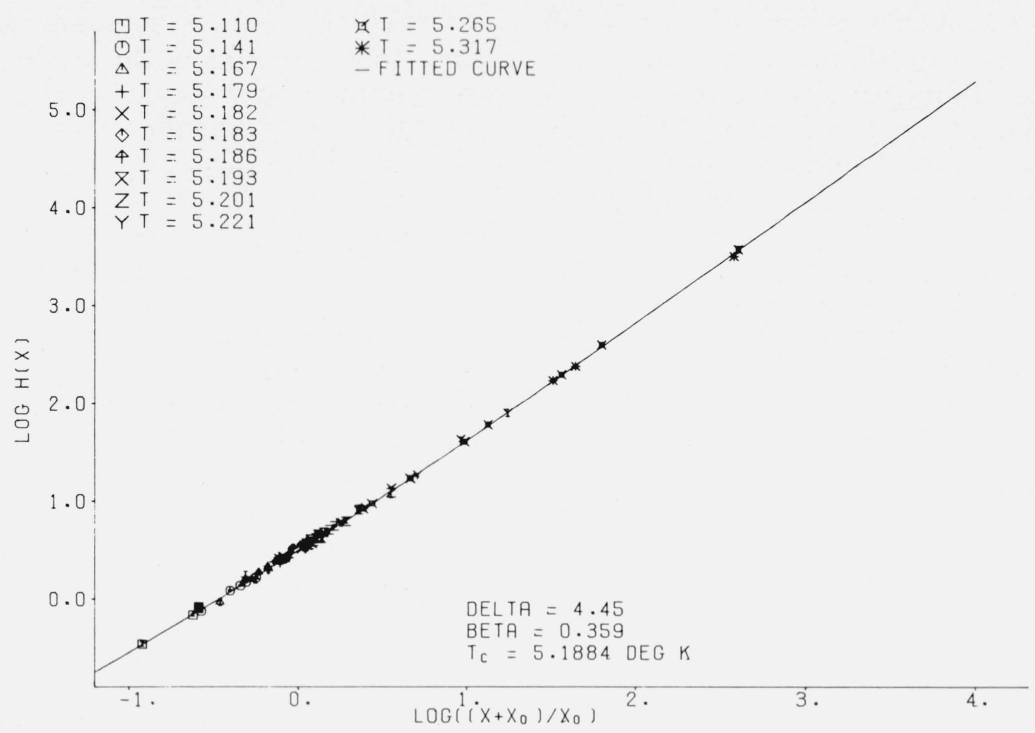

Figure 8A. Log-log plot of $\mathrm{h}(\mathrm{x})$ versus $\left(\mathrm{x}+\mathrm{x}_{0}\right) / \mathrm{x}_{0}$ for $\mathrm{He}^{4}$.

The solid line represents $h(x)$ according to eq (5.3) with parameters given in table 4 . Experimental points are shown with estimated errors in the variable $h(x)$.

RELATIVE DEVIATIONS: HE 4

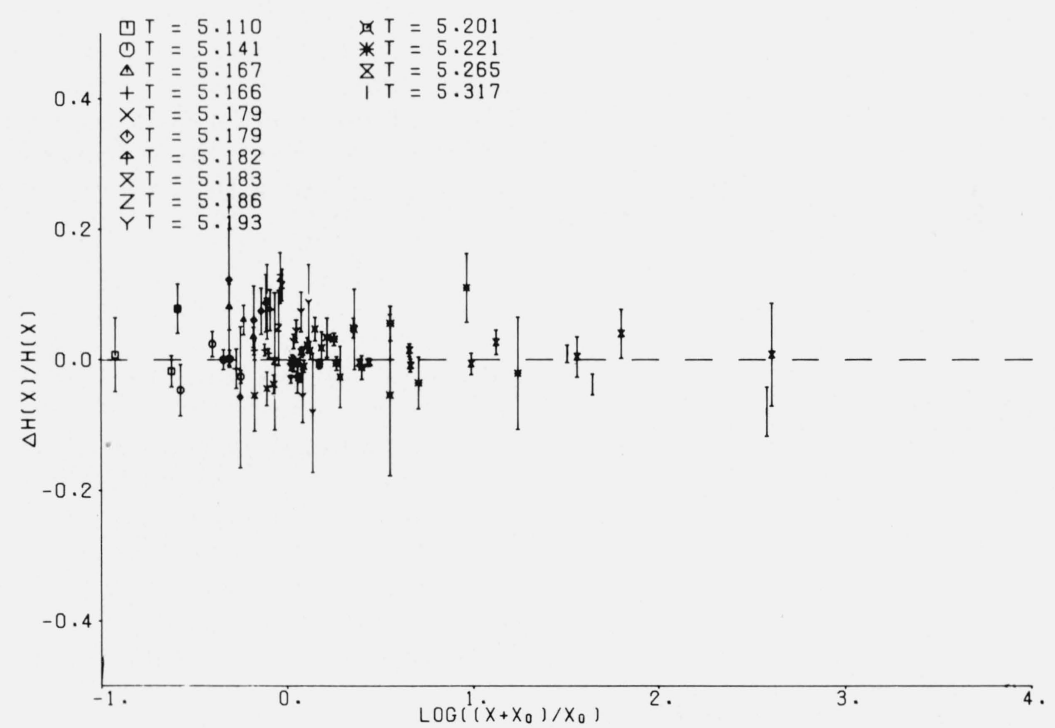

FIGURE 8B. Relative deviation $\Delta \mathrm{h}(\mathrm{x}) / \mathrm{h}(\mathrm{x})=\left[\mathrm{h}_{\exp }(\mathrm{x})-\mathrm{h}_{\text {fitted }}(\mathrm{x})\right] / \mathrm{h}_{\exp }(\mathrm{x})$ versus $\log \left[\left(\mathrm{x}+\mathrm{x}_{0}\right) / \mathrm{x}_{0}\right]$ for $\mathrm{He}^{4}$.

Vertical error bars represent all experimental uncertainties. 
another estimate of the range in which $p$ is linear in $\Delta \rho$ and also in this case important deviations from linearity set in at reduced densities only a few percent away from the phase boundary. The values of $\gamma^{\prime}=1.26$ and $\Gamma^{\prime}=3.8 \times 10^{-2}$ determined by Edwards are in good agreement with our values for $\mathrm{He}^{4}$ shown in table 6 .

TABLE 6. The critical exponents $\gamma$ and $\alpha$ and the calculated parameters which describe the behavior along the preferred curves: critical isochore $\left(\Gamma\right.$ and $\left.\Gamma^{\prime}\right)$ and critical isotherm $(\Delta)$; also the parameters describing the specific heat along the critical isochore $\left[\mathrm{A}^{+}, \mathrm{A}_{\overline{\mathrm{II}}}, \Delta \rho \mathrm{C}_{\mathrm{v}}(-\mathrm{t})^{\alpha}\right]$.

\begin{tabular}{|c|c|c|c|}
\hline & $\mathrm{CO}_{2}$ & $\mathrm{Xe}$ & $\mathrm{He}^{4}$ \\
\hline$\gamma \ldots \ldots$ & $1.26 \pm 0.02$ & $1.26 \pm 0.06$ & $1.24 \pm .04$ \\
\hline$\alpha \ldots$ & 0.04 & 0.04 & 0.05 \\
\hline$\Gamma \ldots$ & $5.26 \times 10^{-2}$ & $5.9 \times 10^{-2}$ & $1.30 \times 10^{-1}$ \\
\hline$\Gamma^{\prime} \ldots$ & $1.19 \times 10^{-2}$ & $1.43 \times 10^{-2}$ & $3.59 \times 10^{-2}$ \\
\hline$\Gamma / \Gamma^{\prime}$. & 4.4 & 4.1 & 3.6 \\
\hline$\Delta \ldots \ldots$ & 2.6 & 3.3 & 3.2 \\
\hline$A^{+} \ldots$ & 4.24 & 3.70 & 1.06 \\
\hline$A_{\mathrm{II}}^{-} \ldots \ldots \ldots \ldots \ldots$ & 5.80 & 4.80 & 1.42 \\
\hline$\Delta \rho C_{v} \cdot(-t)^{\alpha}$. & 39 & 28 & 7.3 \\
\hline
\end{tabular}

We note that the values 0.76 and 0.56 reported for $\Delta$ in ref. [5] actually were the values of $\log \Delta$. We note also that the values for $\Delta \rho C_{v}(-t)^{\alpha}$ reported in ref. [28] correspond to the actual values multiplied by $\alpha$.
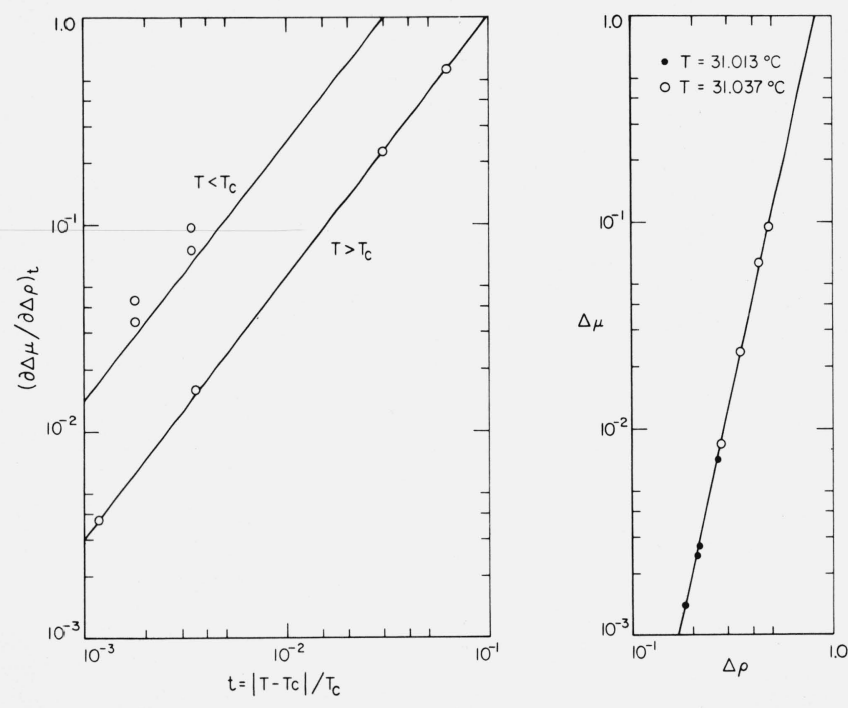

FIGURE 9. Comparison between the experimental results and the prediction of the fit for the compressibility on the critical isochore above $\mathrm{T}_{\mathrm{c}}$, the compressibility on the phase boundary below $\mathrm{T}_{\mathrm{c}}$ $(9 A)$ and the critical isotherm $(9 B)$.

The experimental results for the compressibility are obtained assuming that the $\mu, \rho$ isotherms are linear in $\rho$ in an appreciable range around $\rho_{c}\left(T>T_{c}\right)$ or near the phase boundary $\left(T<T_{c}\right)$. The solid lines represent the prediction from the fit of the $\Delta \mu, \Delta \rho$, $t$ data.

\section{Consistency Checks: Vapor Pressure and Specific Heat}

In section 2 it was shown that the scaled equation of state predicts the nonanalytic behavior of the free energy $a(\rho, t)$ and therefore of the pressure and of the specific heat at constant volume. Thus external consistency checks of the scaling of the $\Delta \mu, \Delta \rho, t$ data are obtained by comparing the anomalies implied for pressure and specific heat with an independent analysis of the directly measured values of $p$ and $C_{v}$.

\subsection{Vapor Pressure and Pressure Along the Critical Isochore at $T>T_{c}$}

The prediction of the scaling law for these quantities has been given in eq (2.19). If the analytic part $A_{0}(T)$ were known, a direct analysis of the pressure would yield information on the terms with $|t|^{2-\alpha}$ and on the value of the exponent $\alpha$ itself. In a relatively small range of $t$ we can assume that a polynomial in $t$ gives an accurate representation of the function $A_{0}(T)$. With a given set of experimental data one can then observe the difference in the fit obtained with a polynomial in $t$ of degree $n$ (perfectly analytic behavior) or with a polynomial of degree $(n-1)$ plus a term $D|t|^{2-\alpha}$. However, the indications are that $\alpha$ is small $(\simeq 0.1)$; thus unless very accurate determinations of the vapor pressure over a not too limited range of temperatures are available, the values of $\alpha$ and $D$ will have large uncertainty, even if the nonanalytic expression gives an improved fit to the data. The product $D \alpha$ is expected to be approximately constant and perhaps somewhat better determined. Vapor pressure data and pressure on the critical isochore above $T_{c}$ should be independently fitted and compared to see whether the analytic part and the values of $\alpha$ are the same. For Xe a reasonable amount of information is available at $T<T_{c}$ in the data of Michels et al. [24], namely, eleven data points between $247.2 \mathrm{~K}$ and $289.2 \mathrm{~K}$. We have fitted these data with a $3 \mathrm{~d}$ degree polynomial and with the nonanalytic expression

$$
\frac{p-p_{c}}{p_{c}}=a t+b t^{2}-a_{\alpha}\left(-x_{0}\right) x_{0}^{\alpha-2}(-t)^{2-\alpha} .
$$

Although the two functions have the same number of adjustable constants, the nonanalytic expression has a standard deviation of a factor 4 smaller for any $\alpha$ between 0.04 and 0.08 . Due to the fact that there are no points very close to $T_{c}$, small variations in $T_{c}$ (and $p_{c}$ ) do not affect the results. Values of the constants $a, b, a_{a}\left(-x_{0}\right) x_{0}^{\alpha-2}$ are given in table 7 . Because of the scarcity of data we did not study the dependence of the parameters on the range.

TABLE 7. Constants of the vapor pressure equation: $\Delta \mathrm{p} / \mathrm{p}_{\mathrm{c}}=\mathrm{at}+\mathrm{bt}^{2}-\mathrm{a}_{\alpha}\left(-\mathrm{x}_{0}\right) \mathrm{x}_{0}^{\alpha-2}(-\mathrm{t})^{2-\alpha}$, for Xe ref. [24] and $\mathrm{CO}_{2}$ ref. [25]

\begin{tabular}{cc|l|l|c|c}
\hline \hline & $\alpha$ & \multicolumn{1}{|c|}{$a$} & \multicolumn{1}{|c|}{$-b$} & $-a_{\alpha}\left(-x_{0}\right) \cdot x_{0}^{\alpha-2}$ & $-\alpha a_{\alpha}\left(-x_{0}\right) x_{0}^{\alpha^{-2}}$ \\
\hline $\mathrm{Xe}$ & 0.04 & $6.02 \pm .01$ & $76 \pm 2$ & $82 \pm 2$ & $3.28 \pm 0.08$ \\
& 0.06 & 6.02 & 47 & 53 & 3.18 \\
& 0.08 & 6.02 & 32 & 38 & 3.04 \\
$\mathrm{CO}_{2}$ & 0.04 & $6.980 \pm .002$ & $85.1 \pm .8$ & $93.8 \pm .7$ & $3.75 \pm .3$ \\
& 0.06 & 6.984 & $51.1 \pm .4$ & $60.1 \pm .5$ & $3.60 \pm .3$ \\
& 0.08 & 6.988 & $34.2 \pm .3$ & $43.4 \pm .4$ & $3.46 \pm .3$ \\
\hline
\end{tabular}


TABLE 8. Parameters describing the behavior of the specific heat along the critical isochore: com varison between values derived (a) from fit of the $\Delta \rho, \Delta \mu, \mathrm{t}$ data, (b) from fit of vapor pressure data, (c) from direct measurement of $\mathrm{C}_{\mathrm{v}}$, (d) our analysis of the experimental $\mathrm{C}_{\mathrm{v}}$ data of ref. $[11]$

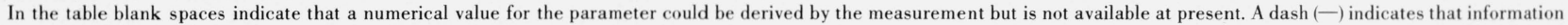
on the parameter cannot be derived from the corresponding analysis.

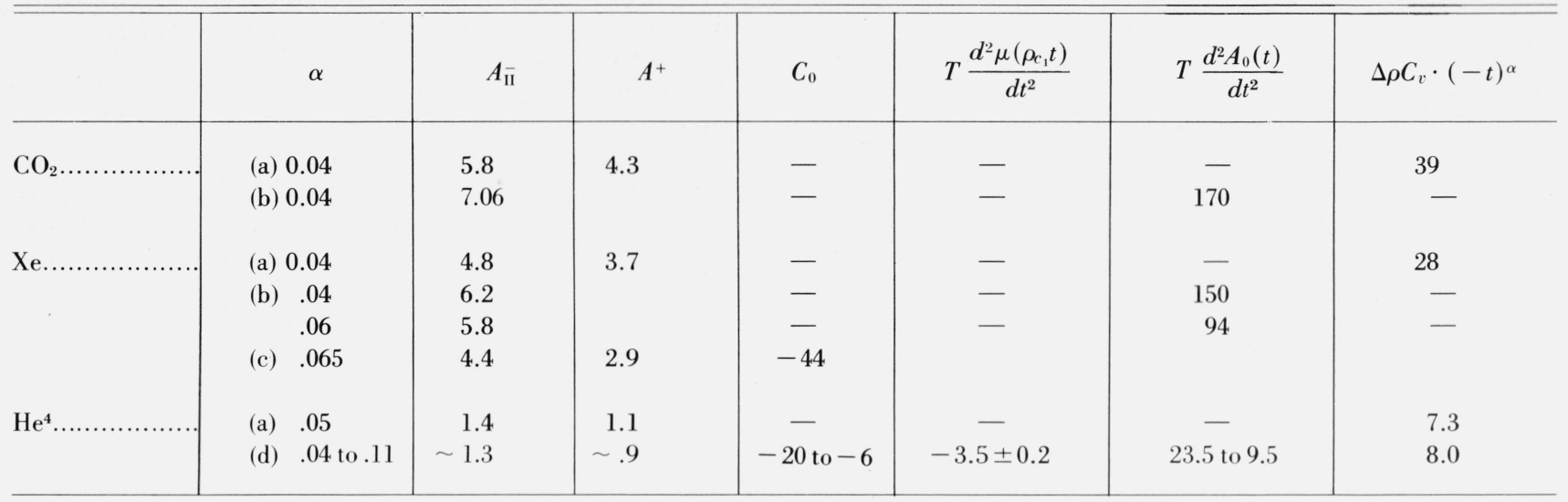

For $\mathrm{CO}_{2}$, precise vapor pressure data have been recently measured [25], with 29 points between $-5{ }^{\circ} \mathrm{C}$ and $31^{\circ} \mathrm{C}$. Here, the data were fitted to a $3 \mathrm{~d}$ degree polynomial and a nonanalytic expression analogous to (9.1). Use of the nonanalytic expression decreases the standard deviation of the fit by a factor 7. $T_{c}, \alpha$ and the range were varied and an optimum fit was obtained for $\alpha$ between 0.04 and 0.10 for the range 4 to $31{ }^{\circ} \mathrm{C}$. From the coefficient of the nonanalytic term, and using the scaling laws eq (2.19) and (2.24), values for $A_{\mathrm{II}}^{-} / \alpha$ are derived and compared with those obtained from scaling the $\Delta \mu, \Delta \rho$ isotherms of Xe and $\mathrm{CO}_{2}$ in table 8 . In view of the large uncertainty in the value of $\alpha$ and the correlation between $\alpha$ and $A_{\mathrm{II}}^{-}$we feel the agreement is reasonable. In the same table, values for $A_{\text {II }}^{-} / \alpha$ from direct measurements of the specific heat are also presented. We will discuss the agreement below.

The constant $b$ in the vapor pressure equation yields an estimate of $-T d^{2} A_{0}(t) / d t^{2}$, which is also given in table 8.

\subsection{Specific Heat}

From the scaling law eq $(2.21)$ it is seen that in the one phase region

$\left[\rho C_{v} / T+\frac{d^{2} A_{0}(t)}{d t^{2}}+\rho \frac{d^{2} \mu\left(\rho_{c}, t\right)}{d t^{2}}\right]|\Delta \rho|^{\alpha / \beta}=-a_{\alpha}^{\prime \prime}(x)$

that is, the quantity on the left-hand side is a function of the variable $x$ only; its properties are related to the properties of $h(x)$. If functional forms of $a_{\alpha}^{\prime \prime}(x)$ and $\frac{d^{2} A_{0}}{d t^{2}}$ could be postulated this relation could be used to check the scaling of data measured at various densities and temperatures. The situation would then be analogous to the scaling of the $\Delta \mu, \Delta \rho$ data, but while in the latter case only one function $h(x)$ had to be chosen, here we have two unknown functions $\frac{d^{2} A_{0}}{d t^{2}}$ and $a_{\alpha}^{\prime \prime}(x)$. In order to avoid making additional assumptions, we decided to analyze only those specific heat data that are close to the critical isochore. For these the scaling laws predict the following behavior:

$$
\begin{array}{ll}
C_{v}=C_{0}+\left(A^{+} / \bar{\alpha}\right) T t^{-\alpha} & t>0 \text { One-phase region } \\
C_{v}=C_{0}+\left(A_{\overline{\mathrm{II}}} / \alpha\right) T|-t|^{-\alpha} & t<0 \text { Two-phase region }
\end{array}
$$

with

$$
C_{0}=-T\left[\frac{d^{2} \mu\left(\rho_{c}, t\right)}{d t^{2}}+\frac{d^{2} A_{0}(t)}{d t^{2}}\right]
$$

assumed to be constant in a small range of $t$. If this asymptotic form is assumed valid over a comparable range of $t$ above and below $T_{c}$, one can determine the values of the parameters $\alpha, \mathrm{C}_{0}, A^{+}, A_{\mathrm{II}}^{-}$by fitting expressions (9.3) to the experimental data; systematic deviations should appear at the same values of $|t|$ on both branches.

Experimental data are available for $\mathrm{He}^{4}$ in the measurements of Moldover [11] and for Xe in the measurements of Edwards, Lipa and Buckingham [26]. We made an analysis of the raw data on helium and obtained the range of values given in table 8. As an example, a plot of $\left(C_{v}-C_{0}\right) T^{-1}$ with $C_{0}=-0.7 \mathrm{~J} / \mathrm{cm}^{3} \mathrm{~K}$ or 15.5 reduced units is given in figure 10 . The data at $T<T_{c}$ are on a straight line of slope $\alpha=0.06$ and the same value of $\alpha$ is compatible with the data at $T>T_{c}$; in the latter case the points with $t<10^{-3}$ may be slightly affected by gravity. From the values of $C_{0}$ and the known value of $T d^{2} \mu\left(\rho_{c}, t\right) / d t^{2}$ values for $d^{2} A_{0} / d t^{2}$ are derived. (Table 8.) The $C_{v}$ data for Xe have been analyzed by the authors [26] according to eq (9.3) with $T$ assumed constant and equal to $T_{c}$. They conclude that any value of $\alpha$ between 0 and .12 gives a reason- 


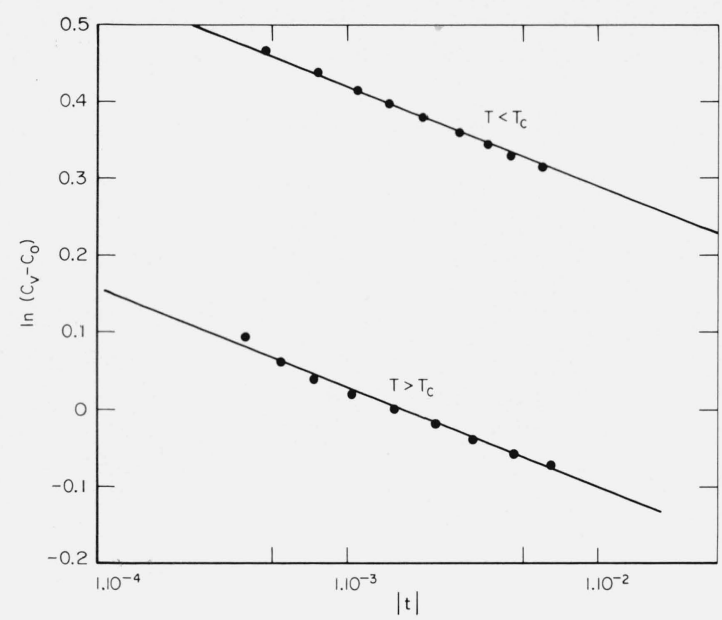

Figure 10. Log-log plot of the experimental data on the specific heat of $\mathrm{He}^{4}$, ref. [11].

In this plot $C_{r}, C_{0}$ are in $\mathrm{J} / \mathrm{cm}^{3} \mathrm{~K} . C_{0}$ has been assumed constant and equal to $-0.7 \mathrm{~J} / \mathrm{cm}^{3}$ $\mathrm{K}$, and $T_{c}=5.1889 \mathrm{~K}$. The solid lines are lines of slope $\alpha=\alpha^{\prime}=0.06$. Similar plots are obtained with $C_{0}$ in the range -0.3 to $-0.9 \mathrm{~J} / \mathrm{cm}^{3} \mathrm{~K}$. The corresponding values of $\alpha$ will range from $\alpha=0.04$ to $\alpha=0.11$.

able fit to the data; their values for the parameters are also listed in table 8 .

The comparison with the values of the same parameters as obtained from the analysis of the $\Delta \mu, \Delta \rho, t$ data is quite good. It should however be mentioned that in the case of helium our value of $\alpha$ does not seem to agree with the value given recently by Moldover by a scaling-law analysis of his data. In appendix I we discuss Moldover's procedure and the possible reason for the disagreement.

In conclusion, there are presently no indications of serious inconsistencies between the scaling-law approach of the equation of state, and the behavior of vapor pressure and specific heat of $\mathrm{CO}_{2}, \mathrm{Xe}$ and $\mathrm{He}^{4}$.

\section{Conclusions}

In applying the scaling laws in their present formulation to the critical region of fluids, the antisymmetry of the chemical potential with respect to the critical density is a prerequisite. We observed this antisymmetry in the experimental data in the critical region of a number of fluids in an appreciable range of density and temperature. As we have pointed out (sec. 3), a corollary of the antisymmetry is the analyticity of $\mu\left(\rho_{c}, T\right)$ at $T_{c}$. This analyticity has been found not to be contradicted by the scarce experimental $C_{v}$ data presently available. Scaling of the experimental $\Delta \mu$, $\Delta \rho, t$ data for $\mathrm{He}^{4}, \mathrm{CO}_{2}$ and $\mathrm{Xe}$ has been shown to be possible within experimental precision in a density range of about \pm 30 percent from critical and for temperatures within -1 percent to +3 percent from critical. A form we propose for the scaling function $h(x)$ fits the data for these three fluids and predicts reasonable values for the anomalous part of $C_{v}$ and of the vapor pressure. The values of the critical exponents determined by the use of the function vary only slightly from substance to substance. There is hardly if any dependence on the quantum parameter $\Lambda^{*}[27]$. The coefficient $E_{1}$ and $E_{2}$ in our equation do not vary appreciably either from substance to substance; thus the deviations from corresponding states are almost completely described by the parameter $x_{0}$, or $B$, alone, which parameter varies considerably from substance to substance.

The present analysis has made it clear that new experiments are needed; in fact, several of the above conclusions are tentative because of lack of sufficiently precise and extensive experimental information. In particular, the fundamental question of the antisymmetry of $\Delta \mu$ should be further investigated. Most useful would be studies of the density profile in the gravity field both above and below $T_{c}$, since this is a direct probe of the $\mu, \rho$ relation. Particular care should be given to avoid vertical temperature gradients. At the same time, the behavior of $d^{2} \mu\left(\rho_{c}, t\right) / d t^{2}$ should be elucidated by precise $C_{v}$ versus $\rho$ measurements in the two-phase region approaching the critical temperature as closely as possible. $C_{v}$ measurements in the one-phase region, particularly at $T_{c}$ and along the two-phase boundary below $T_{c}$, will, in addition, provide better information about the exponents $\alpha$ and $\alpha^{\prime}$. The specific heat measurements should be supplemented with measurements of the pressure on the critical isochore both above and below $T_{c}$ to check whether the divergence of $C_{v}$ is indeed fully explained by the divergence of $\left(\partial^{2} p / \partial t^{2}\right)$. Finally, precise $P V T$ data are needed, in particular near the two-phase boundary. The most useful approach would be to obtain as many of these properties as possible for the same substance.

At the present level of experimental precision the scaling idea, including the assumption of antisymmetry of $\Delta \mu$, and the equation we propose, seem to hold satisfactorily. If, from more refined data, systematic deviations from our function were fol.nd but scaling is still observed, other closed-form expressions for the function $h(x)$ could be considered. For instance, a modification of our function, which could give a better agreement close to the phase boundary, and at the same time move the nonanalyticity at $x=-x_{0}$ to a point $x=-x_{1}\left(x_{1}>x_{0}\right)$ inside the two-phase region, is readily obtained by replacing $\left(\frac{x+x_{0}}{x_{0}}\right)^{2 \beta}$ in eq (5.3) by $\left(\frac{x+x_{1}}{x_{0}}\right)^{2 \beta}$. More precise data could also reveal deviations from scaling in the present density-temperature range. This might lead to a refinement of the definition of the asymptotic range without necessitating fundamental changes in the scaling ideas.

However, should it be found that the property of antisymmetry is not fulfilled, then basic changes in the scaling formulation are needed, for instance by choosing new variables such as a combination of pressure and chemical potential. Within the framework of the scaling laws as presently used, the most significant need, from a phenomenological point of view, is the formulation of the free energy as a function of density and temperature, since the knowledge of the $\Delta \mu, \Delta \rho$, $t$ equation of state by itself yields only a partial description of the critical region. 


\section{Appendix I}

When $\alpha=0$, eq (2.16) and (2.17) must be used to derive expressions for the equation of state in terms of the variables $p, \rho, t$ and for the specific heat.

For the pressure one obtains

$$
\begin{array}{r}
p=\Delta \rho|\Delta \rho|^{\delta-1} h(x)+|\Delta \rho|^{\delta+1}\left[h(x)-a_{0}(x)\right] \\
-h_{2} t^{2} \ln |\Delta \rho|-A_{0}(T)
\end{array}
$$

which gives on the critical isochore:

$$
\begin{array}{r}
p\left(\rho_{c}, t\right)=-A_{0}(T)-\beta G t^{2}-\beta h_{2} t^{2} \ln t \\
p_{v}(T)=-A_{0}(T)-t^{2}\left[a_{0}\left(-x_{0}\right) x_{0}^{-2}-\beta h_{2} \ln x_{0}\right] \\
-\beta h_{2} t^{2} \ln |t|
\end{array}
$$

where $G$ is an undetermined constant and $a\left(-x_{0}\right)$ may be evaluated from the expression for $a_{0}(x)$ valid near $x \simeq 0$ :

$$
\begin{aligned}
a_{0}(x) & =\beta\left\{\frac{1}{2} h_{0}+h_{1} x+F x^{2}\right. \\
& \left.-x^{2} \int_{0}^{x}\left[h(y)-h_{0}-h_{1} y-h_{2} y^{2}\right]|y|^{-3} d y\right\}
\end{aligned}
$$

where $F$ is a constant related to $G$ by

$$
F=G+\int_{0}^{\infty} \frac{d y}{y}\left[\frac{h(y)-h_{0}-h_{1} y}{y^{2}}-\frac{h_{2}}{1+y}\right] .
$$

The nonanalytic part is then composed of two parts, a $t^{2} \ln t$ term with the same coefficient above and below $T_{c}$, and a $t^{2}$ term with different coefficient for $t>0$ or $t<0$.

For the specific heat one obtains for $t>0$

$$
-\frac{\rho C_{v}}{T}=\frac{d^{2} A_{0}}{d T^{2}}+\rho \frac{d^{2} \mu\left(\rho_{c}, t\right)}{d t^{2}}+a_{0}^{\prime \prime}(x)+2 h_{2} \ln |\Delta \rho| .
$$

On the critical isochore:

$$
\begin{gathered}
a_{0}^{\prime \prime}(x)=2 \beta h_{2} \ln x+3 \beta h_{2}+2 \beta G \\
-\frac{C_{v}}{T}=\frac{d^{2} A_{0}}{d T^{2}}+\frac{d^{2} \mu\left(\rho_{c}, t\right)}{d t^{2}}+2 \beta h_{2} \ln t+3 \beta h_{2}+2 \beta G
\end{gathered}
$$

in the two-phase region:

$$
\begin{aligned}
-\frac{\rho C_{v}}{T}=\frac{d^{2} A_{0}}{d T^{2}} & +\rho \frac{d^{2} \mu\left(\rho_{c}, t\right)}{d t^{2}}+2 \beta h_{2} \ln |t| \\
& -2 \beta h_{2} \ln x_{0}+3 \beta h_{2}+2 \frac{a_{0}\left(-x_{0}\right)}{x_{0}^{2}} .
\end{aligned}
$$

Therefore the asymptotic expressions for the specific heat on the critical isochore are

$$
C_{v}=A \ln t+B_{+} \quad t>0
$$

$$
C_{v}=A \ln t+B_{-} \quad t<0
$$

with

$$
\begin{aligned}
\mathrm{A} & =2 \beta h_{2}=\beta h^{\prime \prime}(0) \\
\mathrm{B}_{+} & =3 \beta h^{2}+2 \beta G \\
\overline{\mathrm{B}}_{-} & =3 \beta h_{2}-2 \beta h_{2} \ln x_{0}+2 a_{0}\left(-x_{0}\right) x_{0}^{-2}
\end{aligned}
$$

Except for $\mathrm{B}_{+}$, which contains the undetermined constant $G$, these coefficients can be numerically evaluated from the properties of $h(x)$.

The expression for the jump in the specific heat across the phase boundary is

$$
\Delta \rho C_{v}=\left(\beta / x_{0}\right) h^{\prime}\left(-x_{0}\right) .
$$

In contrast to the case $\alpha>0$ or $\alpha<0$, this discontinuity is independent of the temperature.

Since the equation for the discontinuity in $\rho C_{v}$ when crossing the phase boundary does not involve any unknown constant, the experimental determination of this jump would provide the most direct information about $\alpha^{\prime}$.

\section{Appendix II}

Numerical evaluation of the coefficient for the specific heat.

$A^{+} / \alpha-$ This coefficient is given by the integral

$$
\int_{0}^{\infty} h^{\prime \prime}(y) y^{\alpha-1} d y
$$

where $h^{\prime \prime}(y) y^{\alpha-1}$ diverges near $y=0$.

Assuming $\epsilon$ small but different from 0 the integral between $\epsilon$ and infinity is evaluated by standard numerical integration. For the interval $0-\epsilon, h^{\prime \prime}(y)$ is given by

$$
h^{\prime \prime}(y)=2 h_{2}+6 h_{3} y+12 h_{4} y^{2}+\ldots .
$$

then

$$
\begin{aligned}
\int_{0}^{\epsilon} h^{\prime \prime}(y) y^{\alpha-1} d y=2 h_{2} \frac{\epsilon^{\alpha}}{\alpha} & +6 h_{3} \frac{\epsilon^{\alpha+1}}{\alpha+1} \\
& +12 h_{4} \frac{\epsilon^{\alpha+2}}{\alpha+2}+\ldots .
\end{aligned}
$$

For $\epsilon$ sufficiently small, $\epsilon \leqslant 0.1 x_{0}$, evaluating the first two terms only is sufficient.

$A_{\overline{\mathrm{II}}} / \alpha$-The calculation of this coefficients involves the evaluation of $a_{\alpha}\left(-x_{0}\right)$, which from 2.13 depends on the integral

$$
\int_{0}^{x_{0}}\left(h_{2} y^{2}+h_{3} y^{3}+h_{4} y^{4}+\ldots\right)|y|^{\alpha-3} d|y|
$$

or

$$
\int_{0}^{x_{0}}\left(h_{2}-h_{3}|y|+h_{4}|y|^{2}+. .\right)|y|^{\alpha-1} d|y|
$$


which is of the same form as the integral involved in the evaluation of $A^{+} / \alpha$. The same procedure is then chosen, the integration between 0 and $\epsilon$ is performed using the series expansion and gives a contribution

$$
\epsilon^{\alpha}\left[\frac{h_{2}}{\alpha}-h_{3} \frac{\epsilon}{\alpha+1}+h_{4} \frac{\epsilon^{2}}{\alpha+2}+. .\right]
$$

whereas the integral between $\epsilon$ and $x_{0}$ is evaluated by standard numerical integration techniques.

\section{Appendix III}

Moldover's analysis of his $\mathrm{He}^{4}$ specific heat data on 7 isochores is based on the assumptions that $T\left(d^{2} \mu / d T^{2}\right)_{\rho_{c}}$ is a constant (independent of $T$ ) and that the density independent part of the specific heat possesses a weak temperature dependence:

$$
-T\left[d^{2} \mu\left(\rho_{c}, t\right) / d t^{2}\right]-T\left(d^{2} A_{0} / d T^{2}\right)=a+b t .
$$

The singular part of the specific heat in the onephase region, the term $-T|\Delta \rho|^{-\alpha / \beta} \alpha^{\prime \prime}(x)$ in eq (2.21), is chosen as

$-T|\Delta \rho|^{-\alpha / \beta} a^{\prime \prime}(x)=A|\Delta \rho|^{-\alpha / \beta}\left[1+x|x|^{N-1}\right]^{-\alpha / N}$.

The complete expressions for the specific heat that are used to fit the experimental data are as follows:

$$
\begin{gathered}
\rho C_{v}=D \Delta \rho+a+b t+A|\Delta \rho|^{-\alpha / \beta}\left[1+x|x|^{N-1}\right]^{-\alpha / N}, \\
\text { one-phase region }
\end{gathered}
$$$$
\rho C_{v}=D \Delta \rho+a^{\prime}+b t+A^{\prime}(-t)^{-\alpha^{\prime}},
$$$$
\text { two-phase region }
$$

$D, a, a^{\prime}, \alpha, \alpha^{\prime}, b, N, A$, and $A^{\prime}$ are constants to be determined by the fit.

The assumption AIII.2, allows to analyze simultaneously all $C_{v}$ isochores, which is a very attractive procedure. However as Moldover points out, the assumed functional dependence of $x$ is not in complete agreement with the scaling ideas; the most serious problem (in our opinion) being that it does not have a power series expansion around $x=0$.

With eq (AIII.3) the specific heat data on all isochores scale nicely. The numerical value of the constant $D\left[=-T\left(d^{2} \mu / d T^{2}\right)_{\rho_{c}}\right]$ determined by the fit is in agreement with our value (see III). However, the constants $a$ and $a^{\prime}$ are found to be different $\left(a^{\prime}\right.$ is positive and $a$ is negative) which implies that although the singularity in $C_{v}$ is assumed to be described by the term $|\Delta \rho|^{-\alpha / \beta} a^{\prime \prime}(x)$ in the one phase region and by a term $A^{\prime}(-t)^{-\alpha^{\prime}}$ in the two phase region, an additional jump in $C_{v}$ is present on the phase boundary.

The values of $\alpha^{\prime}$ derived by this analysis, $\alpha^{\prime}=0.16$, is in disagreement with the value $\alpha^{\prime}=0$ reported in his thesis and also with the value $\alpha^{\prime} \sim 0.05$ that we find fitting the data in the two-phase region using (AIII.3) with $b=0$.

The additional jump in $C_{v}$ and the high value of $\alpha, \alpha^{\prime}$ are not in agreement with our scaling analysis of PVT data on $\mathrm{He}^{4}$. Further investigations of the properties of the function (AIII.2), its range of validity and the sensitivity of the values of the parameters to variation in the form of the function are needed.

During the course of this research we have benefited from discussions with and preprints from Dr. R. B. Griffiths, Dr. M. E. Fisher and Dr. L. R. Wilcox. We made use of prepublication results kindly forwarded to us by Dr. M. R. Moldover, Dr. P. R. Roach and Dr. M. H. Edwards.

We acknowledge the enthusiastic support of Dr. M. Klein and Dr. R. D. Mountain.

Mr. J. Gallagher capably handled the computer plotting of several of the figures. Dr. M. Cooper and Dr. M. Barmatz provided constructive criticism.

One of us (M.V.M.) has been partially supported by a NATO grant.

\section{References}

[1] Fisher, M. E., in Critical Phenomena, Proceedings of a Conference, Washington, D.C., 1965, [edited by M.S. Green and J. V. Sengers, National Bureau of Standards Miscellaneous Publication No. 273 (U.S. Government Printing Office, Washington, D.C., 1966)], p. 21; J. Math. Phys. 5, 944 (1964).

[2] Widom, B., J. Chem. Phys. 43, 3898 (1965).

[3] Kadanoff, L. P., Physics 2, 263 (1966); C. Domb and D. L. Hunter, Proc. Phys. Soc. (London) 86, 1147 (1965).

[4] Griffiths, R. B., Phys. Rev. 158, 176 (1967).

[5] Green, M. S., Vicentini-Missoni, M., and Levelt Sengers, J. M. H., Phys. Rev. Letters 18, 1113 (1967).

[6] Michels, A., Blaisse, B., and Michels, C., Proc. Roy. Soc. (London) Ser. A160, 358 (1937).

[7] Michels, A., Lunbeck, R. J., and Wolkers, G. J., Physica, 15 689 (1949), Michels, A., Levelt, J. M. H., and Wolkers, G. J., Physica, 24, 769 (1958).

[8] Roach, P. R., Phys. Rev. 170, 213 (1968)

[9] Yang, C. N., and Yang, C. P., Phys. Rev. Letters 13, 303 (1964).

[10] Barieau, R. E., J. Chem. Phys. 49, 2279 (1968).

[11] Moldover, M. R., and Little, W. A., in Critical Phenomena, Proceedings of a Conference, Washington, D.C., 1965, [edited by Green, M. S., and Sengers, J. V., National Bureau of Standards Miscellaneous Publication No. 273 (U.S. Government Printing Office, Washington, D.C., 1966)]p. 79 and discussion pp. 102-103.

Moldover, M. R.. Ph. D. Thesis, Stanford University 1966; Moldover, M. R., Scaling of Specific Heat Singularity of $\mathrm{He}^{4}$ Near its Critical Point, Phys. Rev. 182, 342 (1969).

[12] Bagatskii, M. I., Voronel, A. V., and Gusak, V. G., Zh. Eksperim. i Teor. Fiz. 43, 728 (1962). [English Transl: Soviet Phys:JETP 16, 517 (1963)]: A. V. Voronel, V. G. Snigirev and Yu. R. Chaskin, Zh. Eksperim. i Teor. Fiz. 48, 981 (1964). [English Transl: JETP 21, 653, (1965)]; A. V. Voronel and Yu. R. Chaskin Zh. Eksperim. i Teor. Fiz. 51, 394 (1966). [English Transl: JETP 24, 263 (1967)].

[13] Amirkhanov, Kh. I., and Kerimov, A. M., Teploenergetika 8, 64 (1963); 9, 61 (1963).

[14] Hill, R. W., and Lounasmaa, O. V., Phil. Trans. A252, 357 (1960).

[15] Habgood, H. W., and Schneider, W. G., Can. J. Chem. 32, 98 (1954).

[16] Wentorf, R. H., J. Chem. Phys. 24, 607 (1956).

[17] Schmidt, E. H. W., Critical Phenomena, Proceedings of a Conference, Washington, D.C., 1965, [edited by M. S. Green and J. V. Sengers, National Bureau of Standards Miscellaneous Publication No. 273 (U.S. Government Printing Office, Washington, D.C., 1966)| p. 197; Straub, J., private communication. 
[18] Kerr, E. C., and Sherman, R. H., Proceedings of the 11th Int. Conf. on Low Temperature Phys., St. Andrews, 1968; p. 236 and R. H. Sherman, private communication.

[19] Brickwedde, F. G., van Dijk, H., Durieux, M., Clement, J. R., and Logan, J. K., J. Res. Nat. Bur. Stands. (U.S.) 64A (1) 1-17 (1960).

[20] Edwards, M. H., Proceedings of the 11th Int. Conference on Low Temperature Physics, St. Andrews, 1968, p. 231.

[21] Hilsenrath, J., Ziegler, G. G., Messina, C. G., Walsh, P. J., and Herbold, R. J., OMNITAB-A Computer Program for Statistical and Numerical Analysis. NBS Handbook 101, 1966; revised edition 1968.

[22] Levelt Sengers, J. M. H., Straub, J., and Vicentini-Missoni, M., to be published.
[23] Wilcox, L. R., and Balzarini, D., J. Chem. Phys. 48, 753 (1968), Balzarini, D., Ph. D. thesis, Columbia University 1968 (unpublished).

[24] Michels, A., and Wassenaar, T., Physica 16, 253 (1950).

[25] Levelt Sengers, J. M. H., and Chen, W. T. (unpublished results); Levelt Sengers, J. M. H., Chen, W. T., and Vicentini-Missoni, M., Bull. Am. Phys. Soc. II, 14, 593 (1969).

[26] Edwards, C., Lipa, J. A., and Buckingham, M. J., Phys. Rev. Letters 20, 496 (1968)

[27] de Boer, J., and Lunbeck, R. J., Physica 14, 520 (1948).

[28] Vicentini-Missoni, M., Levelt Sengers, J. M. H., and Green M. S., Phys. Rev. Letters 22, 309 (1969).

(Paper 73A6-570) 